\title{
Unusual Dynamic Charge Correlations in Simple-Tetragonal $\mathrm{HgBa}_{2} \mathrm{CuO}_{4+\delta}$
}

\author{
B. Yu, ${ }^{1}$ W. Tabis $\odot,{ }^{1,2,3}$ I. Bialo $\odot,{ }^{2,3}$ F. Yakhou, ${ }^{4}$ N. B. Brookes,${ }^{4}$ Z. Anderson $\odot,{ }^{1}$ Y. Tang, ${ }^{1}$ G. Yu, ${ }^{1}$ and M. Greven ${ }^{1}$ \\ ${ }^{1}$ School of Physics and Astronomy, University of Minnesota, Minneapolis, Minnesota 55455, USA \\ ${ }^{2}$ Institute of Solid State Physics, TU Wien, 1040 Vienna, Austria \\ ${ }^{3}$ AGH University of Science and Technology, Faculty of Physics and Applied Computer Science, \\ 30-059 Krakow, Poland \\ ${ }^{4}$ European Synchrotron Radiation Facility, 71 Avenue de Martyrs, CS40220, \\ F-38043 Grenoble Cedex 9, France
}

(Received 4 July 2019; revised manuscript received 13 April 2020; accepted 29 April 2020; published 16 June 2020)

\begin{abstract}
The charge-density-wave (CDW) instability in the underdoped, pseudogap part of the cuprate phase diagram has been a major recent research focus, yet measurements of dynamic, energy-resolved charge correlations are still in their infancy. Such information is crucial in order to help discern the connection between CDW and pseudogap phenomena, and to understand the extent to which charge correlations in general shape the phase diagram. We report a resonant inelastic x-ray scattering study of the underdoped cuprate superconductor $\mathrm{HgBa}_{2} \mathrm{CuO}_{4+\delta}\left(T_{c}=70 \mathrm{~K}\right)$. At $250 \mathrm{~K}$, above the previously established temperature $T_{\mathrm{CDW}} \approx 200 \mathrm{~K}$ that signifies the onset of quasistatic short-range CDW order, we observe significant dynamic correlations that are broadly peaked at about $40 \mathrm{meV}$ and centered at the twodimensional wave vector $\boldsymbol{q}_{\mathrm{CDW}}$. This energy scale is comparable to both the superconducting gap and the low-energy pseudogap. At $70 \mathrm{~K}$, we observe a quasistatic CDW peak at $\boldsymbol{q}_{\mathrm{CDW}}$, but the dynamic correlations around $40 \mathrm{meV}$ remain virtually unchanged, and we identify a new feature: dynamic correlations well above the optic phonon range that are broadly peaked in the $150-200 \mathrm{meV}$ range. A similar energy scale was previously identified in other experiments as a high-energy pseudogap. The observation of three distinct features in the charge response is highly unusual for a CDW system and suggests that charge order in the cuprates is complex. We demonstrate that other single-layer cuprates exhibit approximately the same relative strength of high- to low-temperature energy-integrated charge signal, which points to the universal existence of significant dynamic correlations. Intriguingly, the two energy scales identified here are also comparable to those of the superconducting glue function extracted from other spectroscopic techniques, consistent with a dual charge and magnetic nature of the pairing glue. We further determine the paramagnon dispersion along [1,0], across $\boldsymbol{q}_{\mathrm{CDW}}$, and find it to be consistent with magnetic excitations measured by inelastic neutron scattering. Unlike for some other cuprates, our result points to the absence of a discernible coupling between charge and magnetic excitations.
\end{abstract}

DOI: 10.1103/PhysRevX.10.021059

Subject Areas: Condensed Matter Physics, Strongly Correlated Materials, Superconductivity

\section{INTRODUCTION}

The high- $T_{c}$ cuprates are doped charge-transfer insulators with lamellar structures that feature the quintessential $\mathrm{CuO}_{2}$ plane [1]. At moderate and intermediate hole doping, these complex oxides exhibit a partial depletion of the density of states at the Fermi level (the pseudogap, PG). The PG state is characterized by myriad ordering tendencies, including charge-density-wave (CDW) order with a

Published by the American Physical Society under the terms of the Creative Commons Attribution 4.0 International license. Further distribution of this work must maintain attribution to the author(s) and the published article's title, journal citation, and DOI. modulation direction along the planar $\mathrm{Cu}-\mathrm{O}$ bond and a periodicity of 3-4 lattice units [2-20]. Whereas CDW order competes with superconductivity $[7,15,16]$, dynamic CDW correlations have long been argued to play a pivotal role in shaping the phase diagram [Fig. 1(a)] [21-23]. However, key questions remain unresolved. The mechanism of CDW formation could be related to Fermi-surface nesting (i.e., a reciprocal-space mechanism) [24-26] or to strong electronic correlations that lead to charge separation (i.e., a realspace mechanism) $[2,27,28]$. Furthermore, the relation between CDW correlations and the PG is far from understood, with suggestions that either one is the underlying phenomenon $[20,21,23,29,30]$. The short correlation lengths indicate that disorder might play an important role [31,32], and it is still debated how the CDW in the cuprates 

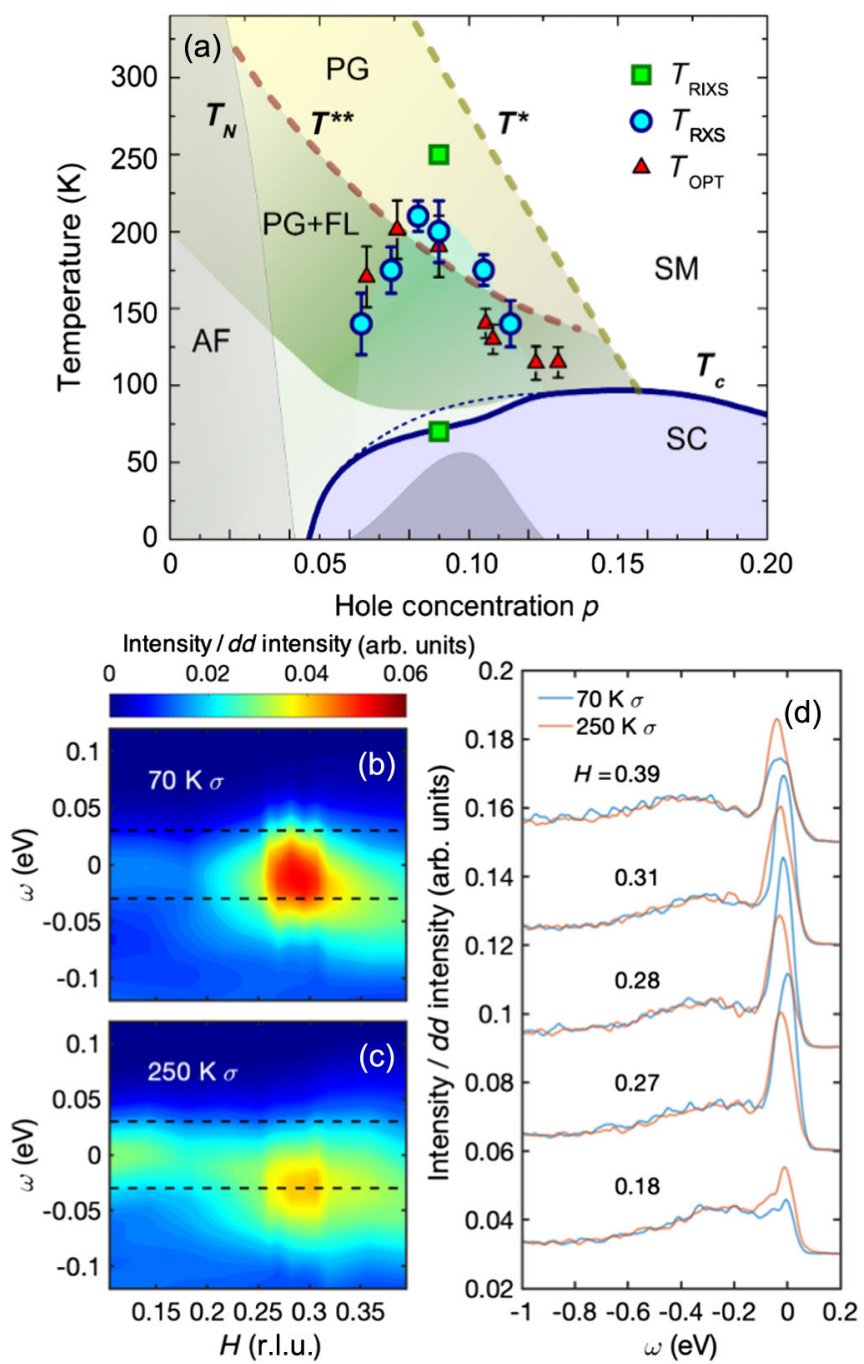

FIG. 1. (a) Hg1201 phase diagram ( $p>0.04)$, extrapolated to $p=0$ based on results for other cuprates [1]. Solid blue line, doping dependence of the superconducting (SC) transition temperature $T_{c}(p)$ [39]. Dark gray region, deviation (not to scale) of $T_{c}(p)$ from estimated parabolic dependence (dashed blue line). $T_{N}$, Néel temperature, marking the transition to antiferromagnetic (AF) order. $T^{*}$, pseudogap (PG) temperature, estimated from deviation from strange-metal (SM) $T$-linear resistivity [40,41]. $T^{* *}$, temperature below which Fermi-liquid (FL) behavior is clearly observed in the PG state [40-43]. $T_{\text {RXD }}$, onset of shortrange CDW correlations estimated from RXS [9,19]. $T_{\mathrm{OPT}}$, characteristic temperature observed in time-resolved optical reflectivity [44]. $T_{\text {RIXs }}$, temperatures at which the $\mathrm{Hg} 1201$ sample $\left(T_{c}=70 \mathrm{~K}, p \sim 0.086\right.$ ) was measured in the present work. (b),(c) Momentum-energy contours of RIXS spectra at 70 and $250 \mathrm{~K}$, obtained with $\sigma$ polarization. Dashed lines, full width at half maximum (FWHM) energy resolution of $60 \mathrm{meV}$. (d) Spectra at select $\boldsymbol{H}$ values, obtained with $\sigma$ polarization; data are vertically shifted for clarity.

becomes static on cooling. Finally, whereas a prominent early theoretical proposal argued in favor of instantaneous pairing [33], ultrafast optical measurements indicate that the interaction in the cuprates is most likely retarded $[34,35]$. Although the relevant bosonic excitations are often argued to be of magnetic origin [36,37], there exists indirect evidence that charge correlations play an important role [29,38].

CDW correlations have been detected in numerous cuprates, primarily via resonant $\mathrm{x}$-ray scattering (RXS) [6-19,45,46], including $\mathrm{HgBa}_{2} \mathrm{CuO}_{4+\delta}(\mathrm{Hg} 1201)[9,19]$. In RXS, the incident photon energy is tuned to the $\mathrm{Cu} L_{3}$ edge to discern modulations of the valence electron density in the $\mathrm{CuO}_{2}$ layers. The scattered photons can be directly measured in energy-integrated mode (conventional RXS), or additionally analyzed by a spectrometer in energy-resolved inelastic mode (RIXS). Conventional RXS has the benefit of relatively short counting times and enabled the efficient exploration of the doping and temperature dependence of CDW correlations. In principle, the charge dynamics can be measured via RIXS, yet until recently, the best available energy resolution was well above $100 \mathrm{meV}$.

We report a RIXS study of the charge dynamics of underdoped $\operatorname{Hg} 1201\left[T_{c}=70 \mathrm{~K}\right.$; see Fig. 1(a)] across $\boldsymbol{q}_{\mathrm{CDW}}$ with energy resolution of $60 \mathrm{meV}$. Although this energy resolution is still an order of magnitude larger than that of, e.g., terahertz experiments $[47,48]$, it is very high for RIXS, and we are able to obtain qualitatively new energy- and momentum-resolved information. $\mathrm{Hg} 1201$ is a single-layer compound, with a simple tetragonal crystal structure and an optimal $T_{c}$ of nearly $100 \mathrm{~K}[29,40-43,49-$ 56]. The model nature of $\mathrm{Hg} 1201$ is exemplified, e.g., by the observations that the normal-state magnetoresistance in the PG state exhibits Kohler scaling [43] and that lowtemperature transport measurements reveal Shubnikovde Haas oscillations due to Fermi-surface reconstruction associated with the CDW order [52,54]. The zero-field CDW order measured via RXS exhibits short in-plane correlation lengths, and there is no evidence for a phase transition $[9,19,32]$. Gaining further understanding of these emergent correlations, and of their connection with the PG phenomenon and the pairing glue, is the primary goal of the present work. The comparatively high onset temperatures, reduced dimensionality, and short length scales suggest that significant dynamic CDW correlations might be present, in analogy with well-known systems such as $\mathrm{NbSe}_{2}$ in the presence of point disorder [12,57-59]. On the other hand, the CDW phenomenology in the cuprates is quite different from conventional systems, as it occurs in the part of the phase diagram that is dominated by the highly unusual PG phenomenon.

RIXS is a unique probe to study such effects. Indeed, at $250 \mathrm{~K}$, well above the temperature $\left(T_{\mathrm{CDW}} \approx 200 \mathrm{~K}\right)$ below which evidence for quasistatic short-range $\mathrm{CDW}$ order was previously observed in Hg1201 via RXS $[9,19]$ and timeresolved optical reflectivity [44], we only discern dynamic charge correlations, with a characteristic energy of about $40 \mathrm{meV}$. At $70 \mathrm{~K}\left(\approx T_{c}\right)$, on the other hand, we observe quasistatic, short-range CDW correlations, and the dynamic response now includes an additional feature in 
the $100-250 \mathrm{meV}$ range that is broadly peaked at about $165 \mathrm{meV}$. The response centered at about $40 \mathrm{meV}$ does not appear to be affected on cooling from $250 \mathrm{~K}$ to $T_{c}$ and may be of mixed charge and phonon character. These two energy scales appear in other observables, and have been associated with both the PG [60] and the superconducting pairing glue [34,61-65]. Moreover, the larger of the two scales has been linked to the strong electronic correlations that cause the charge-transfer gap of the undoped parent insulators [29,56]. Our experiment therefore potentially detects not only CDW correlations but charge correlations in a broader sense. We demonstrate that energy integration of the new RIXS data yields the prior RXS result for $\mathrm{Hg} 1201$, including the high-temperature feature seen at $\boldsymbol{q}_{\mathrm{CDW}}$ in Hg1201 and other cuprates $[9,19]$. This hightemperature feature, which is typically treated as background, is therefore shown to be associated with dynamic correlations. From a comparison with RXS data for other single-layer cuprates at the doping level for which the CDW order is most robust, we find that the strength of the high-temperature dynamic signal is consistently 35\%-50\% of the signal at $\sim T_{c}$. Given the ubiquitous presence of the high-temperature feature in prior RXS data, even at doping levels where CDW correlations are weak, this points to the universal existence of significant dynamic charge (or mixed charge and phonon) correlations throughout the temperature-doping phase diagram. We discuss the possibility that these correlations are rooted in the doped-charge-transferinsulator nature of the cuprates. The present data naturally yield the paramagnon dispersion along $[1,0]$, which we find to be consistent with magnetic neutron scattering results $[53,66]$. The magnetic excitations are nearly unaffected by the unusual charge correlations, consistent with their somewhat different energy scales. However, comparison with the characteristic energy scales of the glue function extracted from optical and Raman spectroscopy points to the distinct possibility that both charge and magnetic excitations contribute to the superconducting pairing glue in the cuprates.

\section{EXPERIMENTAL METHODS}

The measurements were performed with the ERIXS spectrometer at beam line ID32 of the European Synchrotron Radiation Facility (ESRF), Grenoble, France [67]. The incident $x$-ray energy was tuned to the maximum of the $\mathrm{Cu} L_{3}$ absorption peak around $932 \mathrm{eV}$, and the x-ray polarization was set either parallel $(\pi)$ or perpendicular $(\sigma)$ to the scattering plane [68]. The scattered photons were analyzed without considering the final-state polarization. The energy resolution was approximately $60 \mathrm{meV}$, as determined from the full width at half maximum (FWHM) of the nonresonant spectrum of a standard polycrystalline silver sample. In order to prepare a clean, high-quality surface, the $\mathrm{Hg} 1201$ single crystal was cleaved ex situ to reveal a face parallel to the $\mathrm{CuO}_{2}$ planes.
The cleaved surface was uniform and large compared to the beam size, thus minimizing the possibility of the beam drifting off the sample. In order to avoid effects due to beam line energy drifts, the resonant energy was periodically checked via x-ray absorption spectroscopy at the $\mathrm{Cu} L$ edge; see Ref. [68] for a description of the zero-energy determination. Momentum scans were performed by rotating the sample about the axis perpendicular to the scattering plane, and the detector angle was set to $2 \theta=150^{\circ}$. The scattering wave vector is $\boldsymbol{Q}=\boldsymbol{H} \boldsymbol{a}^{*}+K \boldsymbol{b}^{*}+L \boldsymbol{c}^{*}=(H, K, L)$ in reciprocal lattice units, where $a^{*}=b^{*}=1.62 \AA^{-1}$ and $c^{*}=0.66 \AA^{-1}$. $K$ was chosen to be zero, and the scans were taken along $[H, 0, L]$, with $L$ coupled to $H$. Because of the short-range nature of the observed two-dimensional (2D) correlations, the $L$ dependence of the cross section is expected to be negligible, and we quote the 2D reduced wave vector $\boldsymbol{q}=(H, K)$. The intensity of the RIXS spectra was normalized to the integrated intensity of $d d$ excitations, following prior work [69].

\section{RESULTS}

Figures 1(b) and 1(c) show RIXS intensity contour plots at $T=70$ and $250 \mathrm{~K}$, respectively, as a function of $H$ and energy transfer $\omega$ (negative $\omega$ corresponds to energy loss), obtained with $\sigma$-polarized incident $\mathrm{x}$ rays directly from individual energy scans such as those shown in Fig. 1(d) [68]. The maps clearly display signal around $\boldsymbol{q}_{\mathrm{CDW}} \approx$ $(0.28,0)$, consistent with prior RXS work $[9,19]$. Whereas the dominant signal at $70 \mathrm{~K}$ is quasielastic, at $250 \mathrm{~K}\left(>T_{\mathrm{CDW}}\right)$ the response is dynamic and centered in the optic phonon range. Because of the larger phonon contribution, the $250 \mathrm{~K}$ spectra exhibit higher intensity away from $\boldsymbol{q}_{\mathrm{CDW}}$ than the $70 \mathrm{~K}$ data. Although weaker, as expected, charge correlations are also observed in $\pi$ polarization [68].

A more detailed data analysis reveals additional information. Figures 2(a)-2(d) show the momentum dependence of RIXS signal [obtained from energy scans such as those in Fig. 1(d), with $\sigma$ polarization] integrated over the FWHM energy resolution $(60 \mathrm{meV})$ with different energy ranges: quasielastic $(-30,+30) \mathrm{meV}$; inelastic $(-90,-30),(-150$, $-90)$, and $(-210,-150) \mathrm{meV}$. In order to arrive at a systematic estimate of the $q$-integrated signal strength, we perform a heuristic fit of the data to a Gaussian peak, with fixed center $\left(\boldsymbol{q}_{\mathrm{CDW}}\right)$ and width ( 0.075 r.l.u.), plus a concave, polynomial contribution. For Hg1201, the optic phonon range extends to about $75 \mathrm{meV}$ [70-72], and for energy transfers in this range, the "background" contribution invariably includes phonon scattering; for all energy-integration ranges, this smoothly varying contribution is indistinguishable at 70 and $250 \mathrm{~K}$.

The "elastic line" in inelastic scattering mainly originates from scattering off defects and disorder. Because of the high quality of our cleaved sample, the true elastic line is 


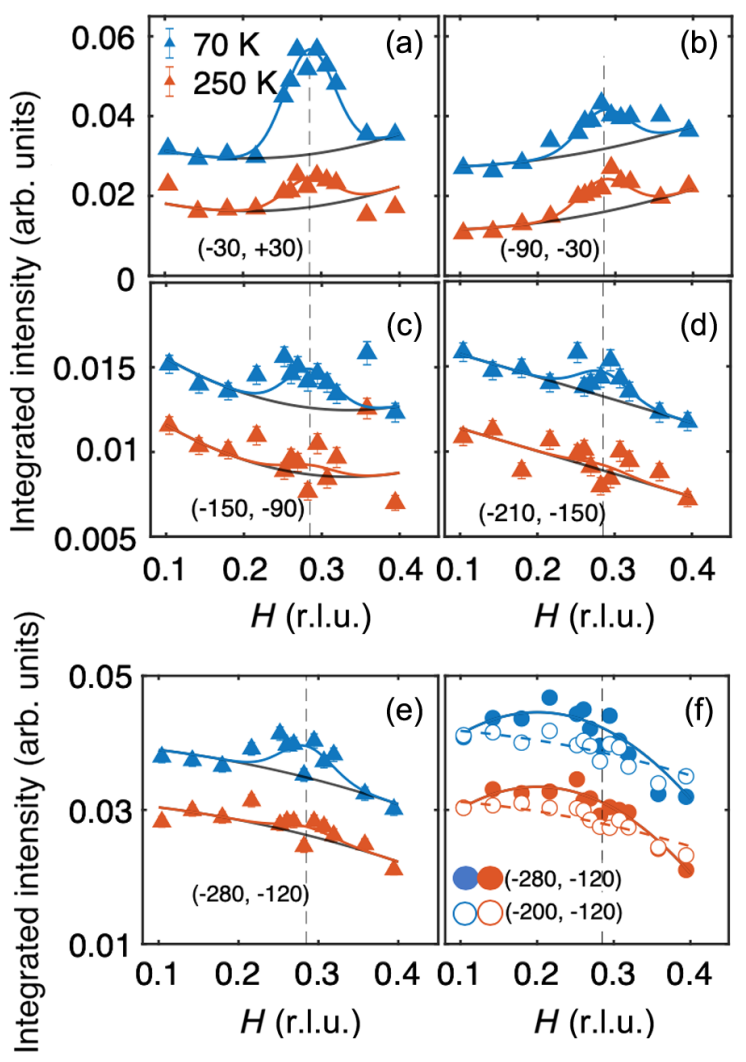

FIG. 2. (a)-(d) $\sigma$-polarized RIXS intensity spectra at 70 and $250 \mathrm{~K}$, integrated over the $60 \mathrm{meV}$ FWHM instrument resolution, centered at $0,60,120$, and $180 \mathrm{meV}$ energy loss. (e) $\sigma$ - and (f) $\pi$-polarized RIXS spectra at 70 and $250 \mathrm{~K}$, integrated over larger energy windows, as indicated. In all cases, the $70 \mathrm{~K}$ curves are vertically shifted for clarity. The black lines in (a)-(e) are polynomial momentum dependences, and the same in each case at low and high temperature. Blue and red lines in (a)-(e) are fits to Gaussian profiles plus a smoothly varying contribution at 70 and $250 \mathrm{~K}$, respectively. Blue and red lines in (f) are guides to the eye. Vertical dashed gray lines indicate $\boldsymbol{q}_{\mathrm{CDW}}$. The errors are one standard deviation (square root of total photon count).

almost impossible to resolve in the raw spectra [Fig. 1(d) herein and Fig. S2 in Ref. [68] ], as the quasielastic signal is always contaminated by other low-energy excitations (mostly phonons). The intensity increase around zero energy and $\boldsymbol{q}_{\mathrm{CDW}}$ due to quasistatic CDW correlations is clearly distinct from the elastic response. Our analysis indicates that the tail of the energy resolution vanishes faster than a Lorentzian function. The increase of the elastic line at small $H$ at $250 \mathrm{~K}$ [Fig. 2(a)] does not increase the inelastic signal [Fig. 2(b)], which further supports our assessment that the high-energy signal is genuine.

Figures 3(a) and 3(b) show the energy dependence of the Gaussian amplitude obtained in this manner for $\sigma$ and $\pi$ polarization, respectively. As expected for charge scattering, the signal is weaker for $\pi$ polarization [68]. The $250 \mathrm{~K}$ results show broad peaks centered at $\sim 40 \mathrm{meV}$ and no evidence for elastic scattering, as the energy dependence of

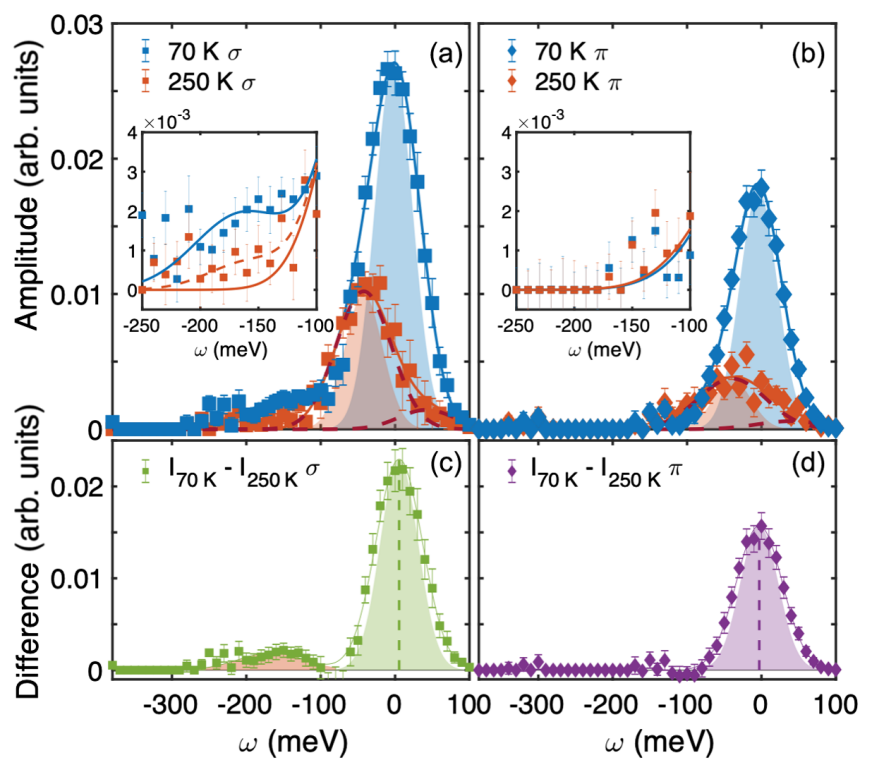

FIG. 3. Energy dependence for (a) $\sigma$ and (b) $\pi$ polarization of fitted Gaussian amplitude from energy-integrated data such as those shown in Figs. 2(a)-2(d). The error bars indicate fit errors. Insets: Enlargement of the range $(-250,-100) \mathrm{meV}$; the dashed red line in the inset of (a) indicates the possibility of nonzero charge scattering in this energy range at $250 \mathrm{~K}$. Blue and red shaded areas indicate the instrument resolution of $60 \mathrm{meV}$ (FWHM). Gaussian fits to Stokes and anti-Stokes scattering at $250 \mathrm{~K}$ (dashed red lines; sum, solid red lines) yield 41(4) and 64(12) $\mathrm{meV}$ for the peak position and intrinsic (deconvoluted) FWHM peak width. Gaussian fits to three peaks at $70 \mathrm{~K}$ (blue lines) capture (1) quasistatic, (2) low-energy ( 40 meV), and (3) high-energy [peak at 163(12) meV, intrinsic width of 77(16) $\mathrm{meV}$ (FWHM)] contributions to the CDW response; the latter is not discerned in $\pi$ polarization, for which the charge response is expected and seen to be weaker [68]. (c),(d) Difference in amplitude between 70 and $250 \mathrm{~K}$ from (a) and (b), respectively, after correcting the $250 \mathrm{~K}$ data for the Bose factor. Green and purple shaded areas indicate the instrument resolution; vertical dashed lines indicate peak centers obtained from fits to Gaussian profiles, which are consistent within error with zero energy transfer. The orange shaded area in (c) indicates the net response centered at $160(6) \mathrm{meV}$.

the amplitude is fully captured by the sum of Stokes and anti-Stokes scattering. The peaks are considerably broader than the $60 \mathrm{meV}$ energy resolution. Assuming a heuristic intrinsic Gaussian profile, we estimate an intrinsic width of at least $50 \mathrm{meV}$ (FWHM) after resolution deconvolution. The RIXS signal may be interpreted either as a single charge mode, with a width that is comparable to its characteristic energy, or as the result of a distribution of charge modes. It is equally well described by a log-normal distribution [68]. We note that our analysis removes phonon contributions that vary smoothly with $H$. Whereas the broad peak in Fig. 1(c) contains low-energy excitations, especially phonons, the extracted intensity at $\sim 40 \mathrm{meV}$ shown in Figs. 3(a) and 3(b) signifies an enhanced charge response at $\boldsymbol{q}_{\mathrm{CDW}}$. As discussed in detail 
below, this enhanced response may in part be due to anomalous phonon scattering.

At $70 \mathrm{~K}$, the dominant charge response is quasistatic. The width of this peak is larger than the energy resolution, consistent with a dynamic contribution at $\sim 40 \mathrm{meV}$ that is unchanged from the response at $250 \mathrm{~K}$. This distinct possibility is highlighted for both polarizations in the (Bose-factor-corrected) intensity difference plots in Figs. 3(c) and 3(d), which reveal resolution-limited elastic peaks centered, within error, at $\omega=0$.

Interestingly, at $70 \mathrm{~K}$ we also observe dynamic charge fluctuations well above the optic phonon range. This is directly seen from Fig. 2, especially Fig. 2(e), where the large binning range $(-280,-120) \mathrm{meV}$ was chosen in order to optimize signal to background. These data, obtained with $\sigma$ polarization, are contrasted in Fig. 2(f) with the equivalent result with $\pi$ polarization, which is more sensitive to magnetic scattering [68]. The convex momentum dependence can be attributed to paramagnons, which become prominent above $\sim 200 \mathrm{meV}$ at $\boldsymbol{q}_{\mathrm{CDW}}$ (see below). This is seen from the comparison in Fig. 2(f) with the result obtained with narrower $(-200,-120) \mathrm{meV}$ integration, which yields an approximately linear background consistent with the $\sigma$-polarization result in Fig. 2(e). From Fig. 3(a), the high-energy charge signal is seen to be peaked at about $165 \mathrm{meV}$; it is not discerned in $\pi$ polarization [Figs. 3(b) and 3(d)] due to the higher background level [proximity to paramagnon excitations; see Fig. 2(f)] and lower expected charge scattering cross section (by a factor of 2 [68]). We note that the $250 \mathrm{~K}$ data in Figs. 2 and 3(a) are consistent with nonzero highenergy charge signal, although with a smaller amplitude and potentially larger momentum width than at $70 \mathrm{~K}$.

Figures 1(d) and 4(a) reveal an additional broad, dispersive peak in the $0.15-0.4 \mathrm{eV}$ range. This feature, which has been observed in a number of cuprates, signifies paramagnon scattering that evolves from well-defined antiferromagnetic excitations in the undoped parent compounds $[73,74]$. These excitations are more prominent in $\pi$ than in $\sigma$ polarization in the present scattering geometry, as expected for magnetic scattering $[68,75,76]$. We extract the paramagnon energy by fitting the raw RIXS spectra to a resolution-limited elastic peak, an effective phonon peak, and a damped paramagnon excitation [68]. Figure 4(b) summarizes our result for the paramagnon dispersion along $[1,0]$ at $70 \mathrm{~K}$. We compare the RIXS data with magnetic neutron scattering data near the antiferromagnetic wave vector for two $\mathrm{Hg} 1201$ samples (one with essentially the same doping level and $T_{c}=71 \mathrm{~K}$ [53], and the other with $p \approx 0.064$ and $\left.T_{c}=55 \mathrm{~K}[66]\right)$ and find that these results are highly consistent and complementary. From a heuristic fit of the combined neutron and x-ray data above $H=$ 0.1 r.l.u. to simple linear spin-wave theory, we obtain an effective nearest-neighbor exchange of 123(3) meV. The relatively large uncertainty and limited data near the zone
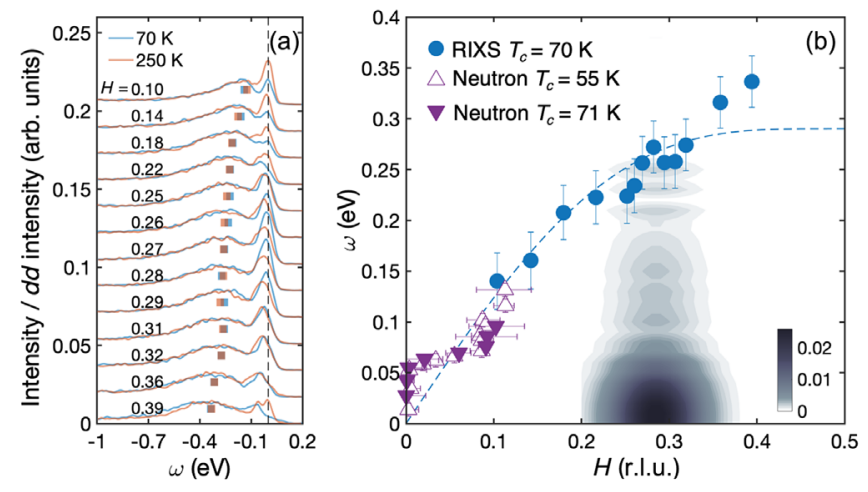

FIG. 4. (a). RIXS spectra for $\mathrm{Hg} 1201$ at numerous wave vectors along $[1,0]$, obtained with $\pi$ polarization. Data are vertically shifted for clarity. Blue and red bars indicate the paramagnon energy obtained from fits to a damped harmonic oscillator (see text and Ref. [68]). (b) Dispersion of magnetic excitations in $\mathrm{Hg} 1201$ measured by RIXS (this work; $p=0.086, T_{c}=70 \mathrm{~K}$ ) and neutron scattering for two $\mathrm{Hg} 1201$ samples: one with nearly the same doping level and $T_{c}=71 \mathrm{~K}$ [53], and the other with a slightly lower doping level of $p \approx 0.064$ and $T_{c}=55 \mathrm{~K}$ [66]. Blue dashed line, heuristic fit to nearest-neighbor spin-wave theory (see text). Error bars for RIXS data are set to $30 \mathrm{meV}$, i.e., half of the FWHM energy resolution. The contour indicates the relative charge intensity at $70 \mathrm{~K}$ [Fig. 3(a)].

boundary prevent a more detailed analysis [77-79]. Overall, the RIXS data for the paramagnon dispersion in $\mathrm{Hg} 1201$ are consistent with prior measurements for other hole-doped cuprates $[68,75,79,80]$.

\section{DISCUSSION}

We observe charge signal in three different energy ranges: (1) a quasistatic response at $70 \mathrm{~K}$, (2) low-energy $(\sim 40 \mathrm{meV})$ signal at 250 and $70 \mathrm{~K}$, and (3) high-energy fluctuations in the 100-250 meV range at $70 \mathrm{~K}$. Below, we first discuss how our observations differ from conventional CDW systems (Sec. IV A). We then consider the possibility that the $40 \mathrm{meV}$ feature signifies a phonon-related effect (Sec. IV B). Next, we discuss our findings within the broader context of the cuprate phase diagram and, in particular, the PG phenomenon (Sec. IV C). Finally, we consider the possible connection between our findings and the superconducting pairing glue (Sec. IV D).

\section{A. Cuprates versus conventional CDW systems}

Analogous to conventional superconductors, conventional CDW systems are described by BCS theory [81]. In clean, weak-coupling systems, macroscopic phase coherence and amplitude formation occur simultaneously at the mean-field transition temperature. In the presence of disorder or at strong coupling, on the other hand, the transition temperature can be significantly depressed, and such systems may exhibit extended PG-like behavior, with 
a gap that persists to high temperature in the absence of long-range order. Such an extended regime has been demonstrated for the canonical CDW compound $2 \mathrm{H}-\mathrm{NbSe}_{2}$ intercalated with $\mathrm{Mn}$ and $\mathrm{Co}$, i.e., in the presence of point disorder [12,57-59].

The PG and charge-order behavior of the cuprates are more complex. These compounds transform from Fermiliquid metal at high doping, with a large Fermi surface that encompasses $1+p$ carriers, to a Mott insulator at zero doping $(p=0)$ via an intermediate state with carrier density $p[40,82,83]$. This evolution involves the localization of exactly one hole per planar $\mathrm{CuO}_{2}$ unit and the concomitant opening of a PG on the "antinodal" parts of the underlying Fermi surface, which leaves ungapped nodal Fermi arcs with carrier density $p$ below $T^{* *}$ (Fig. 1), the lowest of three characteristic PG temperatures (the other two are $T^{*}$ and $T_{\text {hump }}$, with $T^{* *}<T^{*}<T_{\text {hump }}$ ) [29,60]. This Mott-pseudogap phenomenon is qualitatively distinct from the behavior of a canonical CDW system in the presence of strong coupling and/or disorder [59]. The onset of short-range CDW correlations is generally observed at or below $T^{* *}$ (Fig. 1). Associated with the three characteristic PG temperatures are characteristic energy scales, which at the doping level of the present study are about 40-50, 6080 , and $180-220 \mathrm{meV}[60,84]$. The lowest of these scales is the value of the PG near the tip of the arcs [84], whereas the largest scale has been associated with the charge-transfer gap of the insulator, renormalized by the itinerant carriers $[29,56]$. Since the PG formation is gradual and precedes the CDW order [85,86], and because the wave vector $\boldsymbol{q}_{\mathrm{CDW}}$ appears to connect the tips of the arcs $[9,19,54]$, the 40-50 meV PG scale likely sets an upper bound for the CDW gap scale. Given the considerable point disorder exhibited by the cuprates [49], it is perhaps not surprising to observe CDW correlations over an extended temperature range below $T_{\mathrm{CDW}}$, along with a dynamic signature above this nominal ordering temperature, which in this scenario represents a pinning temperature rather than a true phase transition.

However, in this case one would expect a temperaturedependent dynamic scale and a transfer of spectral weight from the dynamic to the static response on cooling, yet this is not what we observe. The difference plots in Figs. 3(c) and 3(d) indicate that the Bose-factor corrected weight of the $\sim 40 \mathrm{meV}$ inelastic contribution is temperature independent, since it cancels out within error for the two temperatures. Equivalently, there seems to be little spectral weight transfer from the $\sim 40 \mathrm{meV}$ dynamic to the quasistatic contribution on cooling, which would not be expected if the CDW were simply pinned below $T_{\mathrm{CDW}}$. Therefore, the quasistatic and $\sim 40 \mathrm{meV}$ contributions likely have different physical origins. In principle, one possibility is an elastic contribution due to the interference of Friedel oscillations around impurities, as suggested theoretically [87] and tested in a quasi-one-dimensional CDW system
[88]. The true underlying CDW signal would then be inelastic even at $T_{c}$, and the CDW correlations would remain dynamic at this temperature. Yet even in this case one would expect a temperature-dependent dynamic CDW signal. A more likely possibility is that the CDW response is quasielastic at all temperatures, whereas the dynamic $\sim 40 \mathrm{meV}$ signal is of a different origin. This conclusion is supported by recent work for $\mathrm{NdBa}_{2} \mathrm{Cu}_{3} \mathrm{O}_{6+\delta}$ (NBCO) [20], which found the amplitude and correlation length of the $\omega \approx 0$ signal to exhibit temperature dependences below $T_{\mathrm{CDW}}$ that point to a gradual buildup of quasistatic correlations and to an underlying phase transition at a temperature below $T_{c}$. As further discussed in the following sections, it is therefore a distinct possibility that the temperature-independent (at least up to $250 \mathrm{~K}$ ) $\sim 40 \mathrm{meV}$ feature at $\boldsymbol{q}_{\mathrm{CDW}}$ is either a direct signature of some other (non-CDW) bosonic charge modes or the result of anomalous phonon scattering, and hence an indirect signature of such modes.

The present data do not allow us to discern if the CDW in the cuprates forms predominantly due to nesting, as in a conventional system, or a real-space mechanism. We can, however, make some inferences at this point. The doping dependence of the CDW wave vector is consistent with a nesting scenario, and quantum oscillation measurements show that the Fermi surface is reconstructed at low temperatures and high magnetic fields, with a resultant electron pocket whose size is consistent with a simple reconstruction scenario [52,54]. However, the quasistatic shortrange CDW correlations observed here at $T_{c}$ clearly do not induce a reconstruction, since transport properties are virtually insensitive to the CDW formation at this temperature [89]. Furthermore, the presence of a significant dynamic component with high onset temperature and the emergent high-energy scale, potentially both signatures of the PG, suggest a strong-coupling scenario, making an underlying real-space mechanism more likely. This is to be expected if the CDW is indeed an emergent phenomenon, since the correlations that cause the cuprate PG appear to be local in real space, as further discussed below. It seems possible that, effectively, both $k$ - and $r$-space effects contribute, which could be related to the deeper question of the existence of a well-defined reciprocal space in a material that is inherently inhomogeneous at the nanoscale $[29,84,85,90]$.

\section{B. Nature of the $40 \mathrm{meV}$ excitation}

We now discuss the possibility that the robust $40 \mathrm{meV}$ excitation signifies the existence of universal dynamic charge correlations in a very large portion of the temperature-doping phase diagram. The $40 \mathrm{meV}$ scale is consistent with superconducting gap and $\mathrm{PG}$ scales for cuprates with a comparable optimal $T_{c}$ [60,84], and with the lower bound of $\sim 20 \mathrm{meV}$ for the gap between the CDW-reconstructed pockets deduced from quantum oscillation experiments for $\mathrm{Hg} 1201$ [54]. This scale lies in the 


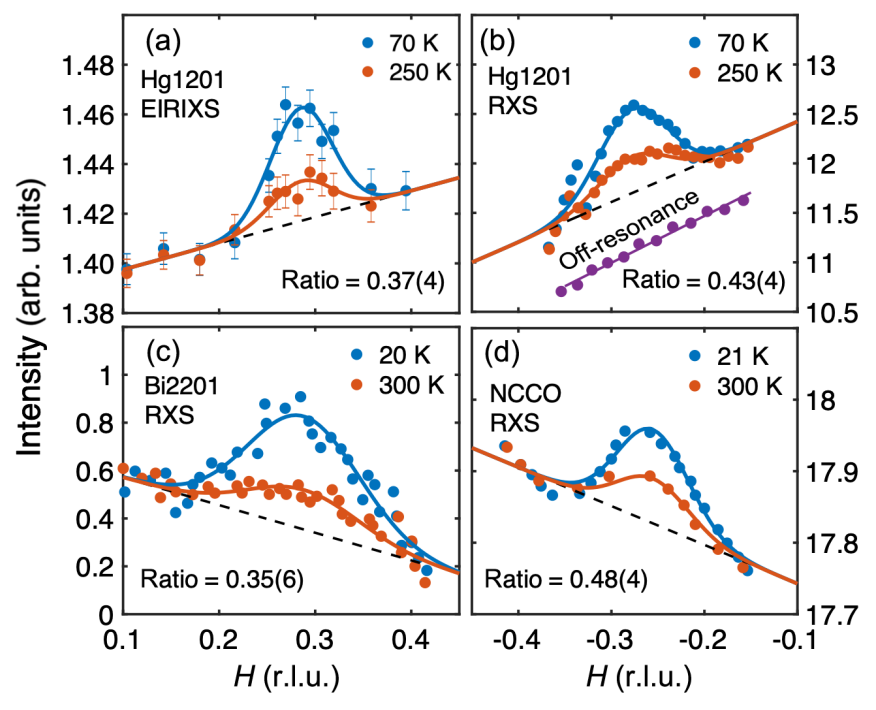

FIG. 5. (a) Energy-integrated RIXS (EIRIXS) data for $\mathrm{Hg} 1201$, integrated over the entire experimental energy range $(-10,2) \mathrm{eV}$, both at $T_{c}$ and $250 \mathrm{~K}$. (b) Prior RXS data for $\mathrm{Hg} 1201$ at the same doping level and temperatures as in (a), along with $70 \mathrm{~K}$ data taken off resonance with an incident photon energy of $929 \mathrm{eV}$, reproduced from Ref. [9]. (c) RXS measurement of $\mathrm{Bi}_{2} \mathrm{Sr}_{2-x} \mathrm{La}_{x} \mathrm{CuO}_{6+\delta}(\mathrm{Bi} 2201, x=0.115)$ at $\sim T_{c}$ and $300 \mathrm{~K}$, reproduced from Ref. [8]. (d) RXS results for superconducting electron-doped $\mathrm{Nd}_{2-x} \mathrm{Ce}_{x} \mathrm{CuO}_{4}(\mathrm{NCCO}, x=0.145)$ at $\sim T_{c}$ and $300 \mathrm{~K}$, reproduced from Ref. [18]. In all cases, the hightemperature data are vertically shifted for comparison with the low-temperature result. Black dashed lines, assumed linear background. Solid lines, fits to Gaussian peak plus assumed linear background. Estimates of relative peak amplitudes are indicated in all panels.

optic phonon range and, in principle, the observed excitation might signify phonon scattering due to enhanced electron-phonon coupling, and thus be an indirect signature of significant dynamic charge correlations. Although there is no evidence for anomalous phonon scattering at $\boldsymbol{q}_{\mathrm{CDW}}$ off resonance [see Fig. 5(b) for a prior RXS result for Hg1201 [9] ], anomalous scattering involving valence electrons could be significantly enhanced at the $\mathrm{Cu} L$ edge. In hole-doped $\mathrm{Bi}_{2} \mathrm{Sr}_{2} \mathrm{CaCu}_{2} \mathrm{O}_{8+\delta}$ (Bi2212) [46], charge correlations were identified up to about $60 \mathrm{meV}$. These correlations were suggested to be dispersive and associated with strong Fano interference at the intersection with the $\mathrm{Cu}-\mathrm{O}$ bond-stretching phonon. At low temperature, a gradual phonon softening near $\boldsymbol{q}_{\mathrm{CDW}}$ was observed, with a minimum energy of 40-45 meV and an intrinsic width of 30-40 meV (FWHM). In $\mathrm{Hg} 1201$, at $\boldsymbol{q}_{\mathrm{CDW}}$, the $\mathrm{Cu}-\mathrm{O}$ bond-stretching phonon lies in the 55-60 meV range, whereas the $\mathrm{Cu}-\mathrm{O}$ bond-bending phonon lies in the 40 $45 \mathrm{meV}$ range [70-72]. It is therefore possible that a similar anomaly involving predominantly the bond-bending phonon is present in $\mathrm{Hg} 1201$. We also note that a recent RIXS study of $\mathrm{La}_{1.8-x} \mathrm{Eu}_{0.2} \mathrm{Sr}_{x} \mathrm{CuO}_{4+\delta}$ suggests significant electron-phonon coupling for the bond-stretching mode around
$\boldsymbol{q}_{\mathrm{CDW}}$. This coupling is observed at high temperature, in the absence of CDW order, and seen to further increase in the ordered state [91].

Alternatively, the $40 \mathrm{meV}$ feature could be a direct signature of charge fluctuations, for a number of reasons. First, unlike the result for Bi2212 [46], our data indicate no change in the $\sim 40 \mathrm{meV}$ charge response (amplitude, characteristic energy, and width) between high and low temperatures (Fig. 3). Second, we estimate the intrinsic width of the excitation to be at least $50 \mathrm{meV}$ (FWHM) (Fig. 3). This value is considerably larger than what might be associated with phonon linewidth broadening, and about 50\%-100\% larger than the width of the peak ascribed to anomalous phonon scattering in Bi2212 [46]. Third, we note that conventional RXS measurements generally reveal a local intensity maximum at or near $\boldsymbol{q}_{\mathrm{CDW}}$ already at high temperatures $[6,8,9,13,18]$. This feature is commonly regarded as a temperature-independent background and subtracted from the low-temperature data to extract the CDW signal, although it also has been associated with charge correlations $[18,46]$, as clearly supported by our RIXS results in Figs. 2 and 3. By integrating over the whole energy range $(-10,2) \mathrm{eV}$ of our experiment, we demonstrate in Figs. 5(a) and 5(b) that our RIXS data are highly consistent with the previous RXS study of $\mathrm{Hg} 1201$ at the same doping level [9]. As seen from Figs. 2 and 3, and further discussed in Ref. [68], most of the signal at or near $\boldsymbol{q}_{\mathrm{CDW}}$ originates from within the $(-0.1,0.1) \mathrm{eV}$ energy range. In Figs. 5(c) and 5(d), we reproduce the corresponding RXS results for $\mathrm{Bi}_{2} \mathrm{Sr}_{2-x} \mathrm{La}_{x} \mathrm{CuO}_{6+\delta}(\mathrm{Bi} 2201, x=$ 0.115) [8] and electron-doped $\mathrm{Nd}_{2-x} \mathrm{Ce}_{x} \mathrm{CuO}_{4}$ (NCCO, $x=0.145$ ) [18], respectively. Like Hg1201, both of these cuprates are single- $\mathrm{CuO}_{2}$-layer compounds. The chosen doping levels are approximately those at which the CDW phenomenon is most robust. Remarkably, the ratios of the intensity amplitudes at or near $T_{c}$ and $T_{\mathrm{CDW}}$ lie in the narrow 35\%-50\% range for all three single-layer cuprates. Along with the insight gained here for Hg1201 (Figs. 2 and 3), this points to the existence of universal dynamic correlations, in addition to quasistatic correlations that emerge below $T_{\mathrm{CDW}}$. Since phonon dispersions vary among the cuprates, and because any anomalous electron-phonon coupling can be expected to vary as well, this appears to rule out the possibility that the $40 \mathrm{meV}$ feature we observe for $\mathrm{Hg} 1201$ is dominated by anomalous phonon scattering. Finally, we note that there exists indirect evidence for collective charge modes from neutron and x-ray scattering experiments of phonon anomalies throughout the superconducting doping range $[92,93]$.

The most likely scenario is that there exist both types of contributions. This is supported by a time- and frequencyresolved optical spectroscopy measurement of optimally doped Bi2212 that revealed bosonic modes of predominantly electronic character in the optic phonon range, along with a significant strongly coupled phonon contribution 
and a small lattice contribution [34]. The above observations point to universal dynamic correlations that extend to high energy and temperature, and that have little temperature dependence between room temperature and $T_{c}$. Moreover, given that prior RXS measurements have revealed a local intensity maximum at or near $\boldsymbol{q}_{\mathrm{CDW}}$ even at high and low doping levels where there is no evidence for quasistatic, low-temperature CDW correlations $[45,68]$, we conclude that there exist universal dynamic correlations in a very large portion of the temperature-doping phase diagram. As discussed in Sec. IV D, these robust dynamic correlations therefore appear to be a precursor of the quasistatic CDW correlations that gradually develop below the doping-dependent temperature $T_{\mathrm{CDW}}$ in a relatively narrow portion of the phase diagram, and that ultimately cause Fermi-surface reconstruction at very low temperatures, once superconductivity is suppressed with a $c$-axis magnetic field.

\section{Comparison with other results for the cuprates}

RIXS measurements of high-energy charge correlations above the optic phonon range have been reported so far only for electron-doped NCCO, where they persist up to approximately $0.4 \mathrm{eV}$ [94]. Figures 2 and 3 demonstrate for $\mathrm{Hg} 1201$ at the investigated (hole) doping level that the full dynamic response peaked at $\boldsymbol{q}_{\mathrm{CDW}}$ is captured by integrating up to about $0.3 \mathrm{eV}$. We estimate that at least $20 \%$ of the dynamic charge response at $70 \mathrm{~K}$ originates from above the optic phonon range [68].

A recent RIXS study of hole-doped NBCO [20] found dynamic charge correlations to persist to high temperatures (above $T^{*}$ ). The experiment was performed with $40 \mathrm{meV}$ (FWHM) energy resolution, and the data were analyzed under the assumption that phonon contributions at $\mathbf{q}=$ $(H, 0)$ can be removed by subtracting data obtained at the same $q$ along $\mathbf{q}=(H, H)$, and by considering intensity differences for data obtained at different temperatures. Dynamic energy scales of $15 \mathrm{meV}$ (at 150 and $250 \mathrm{~K}$ ) and $7 \mathrm{meV}(90 \mathrm{~K})$ were deduced for an optimally doped sample with $T_{c}=90 \mathrm{~K}(p \approx 0.17)$, whereas scales of $6 \mathrm{meV}(150$ and $250 \mathrm{~K})$ and $3 \mathrm{meV}(90 \mathrm{~K})$ were obtained for an underdoped sample with $T_{c}=60 \mathrm{~K}(p \approx 0.17)$. The high-temperature signal was found to exhibit short spatial correlations and to be centered at a somewhat smaller wave vector than the quasistatic CDW response. Our data for Hg1201 are overall consistent with these findings, but do not allow us to discern an additional $\sim 10 \mathrm{meV}$ dynamic signal due to the relatively large energy resolution of $60 \mathrm{meV}$ (FWHM) of our experiment. In turn, the NBCO data are consistent with our observation of significant dynamic correlations at higher energies [68].

The large $\sim 165 \mathrm{meV}$ energy scale identified here for hole-doped $\mathrm{Hg} 1201$ is consistent with the high-energy PG ("hump") scale seen in other observables [29,60], including the midinfrared peak seen in optical spectroscopy experiments [95,96]. A recent phenomenological model rooted in the dual empirical observations of a universal transport scattering rate [41,97] and inherent inhomogeneity $[55,98,99]$ associates this scale with the chargetransfer gap at zero doping and with the delocalization of one hole per planar $\mathrm{CuO}_{2}$ unit above $T^{* *}$ (Fig. 1) [29,56]. For $\mathrm{Hg} 1201$, at $p \approx 0.09$, the mean (de)localization gap and (Gaussian) gap distribution width that best capture the transport data are $\sim 180$ and $\sim 60 \mathrm{meV}$ (FWHM) $[29,56]$, respectively, consistent with the characteristic CDW scale of 163(12) meV and width of 77(16) meV established in the present work [Figs. 3(a) and 3(c)].

The gradual delocalization of one hole per $\mathrm{CuO}_{2}$ unit is a large effect that clearly manifests itself in, e.g., the evolution of the Fermi surface with doping and the strong temperature and doping dependence of the Hall number $[29,56,83]$. In contrast, the CDW order involves a relatively small fraction of a hole per unit cell. While x-ray scattering experiments cannot provide the absolute CDW amplitude, a value of about 0.03 hole per $\mathrm{CuO}_{2}$ unit was estimated for $\mathrm{La}_{2-x} \mathrm{Ba}_{x} \mathrm{CuO}_{4}$ [27]. A separate, consistent estimate can be obtained from NMR: from the universal relation between oxygen hole content and nuclear quadrupole resonance (NQR) frequency [100] and the ${ }^{17} \mathrm{O} N Q R$ line broadening in the CDW phase [12], we estimate 0.028 hole in the $\mathrm{Cu}$ $3 d$ orbital for $\mathrm{Hg} 1201$ [101]. Although $\sim 0.03$ hole per $\mathrm{CuO}_{2}$ unit is a relatively large fraction of the nominal doping level $p$, it is small compared to 1 . It therefore seems likely that the CDW phenomenon in the cuprates is a secondary, emergent phenomenon related to the strong correlations that underlie the hole localization. This is supported by STM evidence for a qualitative change in the CDW form factor of Bi2212 at a characteristic scale comparable to the PG scale [30]. Furthermore, it is known that the cuprates are intrinsically inhomogeneous, with local gaps that vary at the nanoscale and persist well above the PG temperature $[85,86]$. Puddles of localized charge that sustain significant dynamic correlations thus may already exist outside of the nominal PG region [above $\left.T^{* *}(p)\right]$. This could account for the present observation for $\mathrm{Hg} 1201$ at $250 \mathrm{~K}$ and for the similar result for NBCO [20]. The relevant wave vector would span the antinodal regions and hence be close to, but smaller than, $q_{\mathrm{CDW}}$.

We have argued that the high-temperature peak at or near $\boldsymbol{q}_{\mathrm{CDW}}$ seen universally in the cuprates is dominated by dynamic fluctuations, which for $\mathrm{Hg} 1201$ at the studied doping level have a characteristic energy of $\sim 40 \mathrm{meV}$. Although not apparent from the data summarized in Fig. 5, there exists evidence from RXS that dynamic correlations centered at or near $\boldsymbol{q}_{\mathrm{CDW}}$ extend throughout a much larger portion of the Brillouin zone, with real-space correlations no larger than 1-2 lattice constants and little doping and temperature dependence (unlike the quasistatic CDW signal). This is perhaps most clearly seen from RXS measurements of $\mathrm{YBa}_{2} \mathrm{Cu}_{3} \mathrm{O}_{6+\delta}$ [45], for which we 
estimate the approximately doping-independent 2Dintegrated strength of the high-temperature signal to be at least an order of magnitude larger than the strongly temperature-dependent quasistatic CDW signal (at the doping level where CDW correlations are strongest) [68], consistent with a separate estimate based on the recent RIXS data for NBCO [20]. In other words, whereas it appears from data such as those in Fig. 5 that the energyintegrated high-temperature signal is about $35 \%-50 \%$ of the low-temperature response, it may in fact be dramatically larger. This signal might be a direct signature of the fluctuations associated with the localized hole $[29,56]$, and the characteristic wave vector may correspond to the distance between the antinodal regions of the Fermi surface on which the PG develops, and hence be somewhat smaller than $q_{\mathrm{CDW}}$. The 2D-integrated strength of the signal is still considerably smaller than what would be expected if it were fully associated with the localization of one hole per $\mathrm{CuO}_{2}$ unit: naively, given that the CDW amplitude corresponds to about 0.03 hole per $\mathrm{CuO}_{2}$ unit, one might expect this signal to be $(1 / 0.03)^{2} \sim 10^{3}$ times larger than the quasistatic CDW response. It is possible that there exists a considerable incoherent (local in real space) contribution to the charge response up to $0.3 \mathrm{eV}$ and even higher energies that is not captured by RXS and RIXS experiments. Similarly, the $\sim 165 \mathrm{meV}$ scale might not be seen at $250 \mathrm{~K}$ in our experiment because the high-energy charge fluctuations are incoherent at high temperatures. We note that recent momentum-resolved electron-scattering measurements of the charge fluctuations in Bi2212 revealed a featureless continuum up to $\sim 1 \mathrm{eV}$ [102] for a wide range of hole concentrations, from underdoped to overdoped, consistent with the existence of significant local excitations.

The above considerations are supported by neutron and Raman scattering results. First, we note that evidence for an underlying collective charge mode has been deduced from anomalies in the $\mathrm{Cu}-\mathrm{O}$ bond-stretching vibration in the 65-85 meV range single-layer $\mathrm{La}_{2-x} \mathrm{Sr}_{x} \mathrm{CuO}_{4}$ (LSCO) in the superconducting doping range [93]. An anomalous linewidth broadening was observed in the entire momentum range from the $2 \mathrm{D}$ zone center to the $2 \mathrm{D}$ zone boundary at $(0.5,0)$ r.l.u. Given the large intrinsic width of the $\sim 40 \mathrm{meV}$ feature in $\mathrm{Hg} 1201$, these findings for LSCO are consistent with anomalous electron-phonon coupling and a potentially universal spectrum of bosonic charge modes. Second, the broad $\sim 165 \mathrm{meV}$ feature seen in our experiment appears to be related to features seen in Raman spectra with $B_{1 g}$ [103] and $B_{2 g}$ symmetry [104]. Raman scattering probes large portions of the Brillouin zone, with distinctly different form factors in $B_{1 g}$ and $B_{2 g}$ symmetry. In $B_{1 g}$ symmetry, a broad feature with characteristic energy of $\sim 200 \mathrm{meV}\left(\sim 1700 \mathrm{~cm}^{-1}\right)$ was identified for a $\mathrm{Hg} 1201$ sample with a slightly larger doping level ( $p \approx 0.11, T_{c}=77 \mathrm{~K}$ ) than in the present work and associated with the two-magnon excitations of the undoped antiferromagnetic parent compounds. We note that both paramagnon and charge excitations might contribute to the $B_{1 g}$ response. While a clear temperature dependence was observed below $\sim T^{*}$, this feature was seen to persist to higher temperatures [103]. In $B_{2 g}$ symmetry, a feature of width $\sim 60 \mathrm{meV}$ centered at $\sim 150 \mathrm{meV}\left(\sim 1250 \mathrm{~cm}^{-1}\right)$ was observed for $\mathrm{Hg} 1201$ with $T_{c}=72 \mathrm{~K}$, i.e., at nearly the same doping level as the present study, and interpreted as a CDW energy scale [104]. These values are remarkably close to the intrinsic width of 77(16) $\mathrm{meV}$ (FWHM) and peak of 163(12) $\mathrm{meV}$ that we extract and associate with charge fluctuations (Fig. 3).

\section{Dynamic correlations and pairing glue}

We now discuss the possibility that the dynamic charge and magnetic scales observed here correspond to features in the bosonic pairing glue function deduced from other spectroscopic techniques. Based on theoretical considerations, it has been argued that incommensurate charge correlations might be the cause of the $d$-wave pairing in the cuprates, possibly in conjunction with antiferromagnetic spin fluctuations $[21,105]$. While RIXS does not measure the glue function directly, it is a probe of both charge and magnetic excitations and can therefore give insight into the excitations and energy scales relevant for pairing.

In an early optical conductivity study, it was suggested that deviations of the in-plane conductivity from the conventional Drude form may originate from coupling to a bosonic mode [106]. Subsequent optical spectroscopy efforts to extract the pairing glue revealed a robust peak around 50-60 meV and a second feature in the 100$300 \mathrm{meV}$ range for numerous cuprates, including optimally doped Hg1201 [34,61-63]. Given that optical spectroscopy yields an average over the Brillouin zone, whereas our RIXS experiment focuses on the vicinity of $\boldsymbol{q}_{\mathrm{CDW}}$, this result is consistent with an interpretation of our data in terms of charge modes that contribute to the pairing glue. This is demonstrated in Fig. 6, which compares the glue function extracted at $100 \mathrm{~K}$ for $\mathrm{Hg} 1201\left(T_{c}=97 \mathrm{~K}\right)$ with our RIXS result for the dynamic response at $T=T_{c}=70 \mathrm{~K}$. As noted in Sec. III, the results in Figs. 3(a) and 3(b) are equally well captured by intrinsic heuristic Gaussian and log-normal functional forms, and the latter is used for the comparison.

Also shown in Fig. 6 is our result for the energy dependence of the strength of the paramagnon response at $70 \mathrm{~K}$ (obtained from the data in Fig. 4), as well as the momentum-integrated, local dynamic magnetic susceptibility obtained from neutron measurements near the antiferromagnetic wave vector for a sample with nearly the same doping level [53] [see also Fig. 4(b)]. Assuming azimuthal symmetry, the former is proportional to the $2 \mathrm{D}$ momentumintegrated paramagnon strength. The neutron data are available in absolute units, and the RIXS paramagnon result is scaled to match the neutron data just below $100 \mathrm{meV}$. The RIXS data for the charge response, in turn, are scaled up by a factor of 10 for ease of comparison and, 


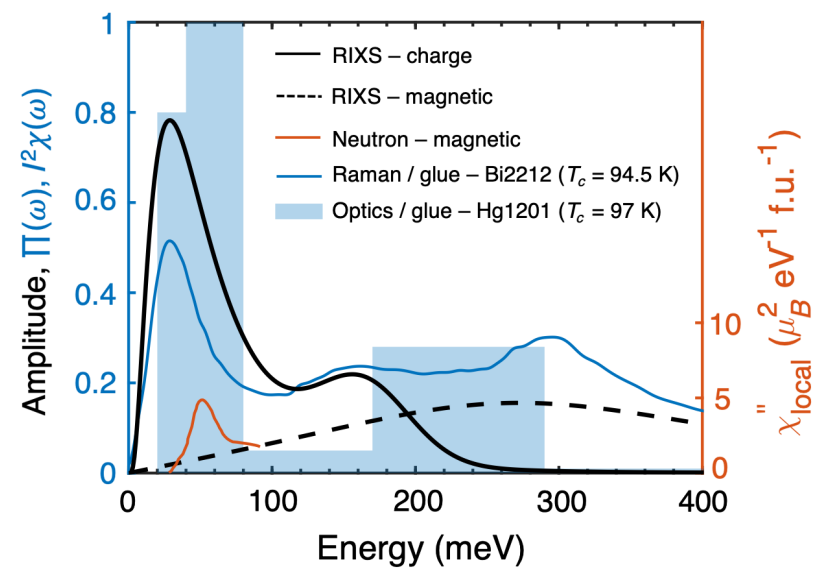

FIG. 6. Comparison of RIXS results for dynamic charge and paramagnon response in $\mathrm{Hg} 1201$ with estimates for the superconducting pairing glue. The black solid and dashed lines are the amplitudes of the charge and magnetic excitations in $\mathrm{Hg} 1201$ at $T=T_{c}=70 \mathrm{~K}$ from the present work. As discussed in the text, the charge signal is multiplied by a factor of 10 . The red solid line indicates the local magnetic susceptibility of $\mathrm{Hg} 1201$ measured by neutron scattering at the nearly same doping level [53]. The blue shaded area is the bosonic glue function extracted from optical measurements of optimally doped $\mathrm{Hg} 1201\left(T_{c}=97 \mathrm{~K}\right)$ at $100 \mathrm{~K}$ [63]; in this work, the glue function was not extracted for underdoped $\mathrm{Hg} 1201$, but it was established from analysis of other cuprates that the two characteristic energy scales are rather robust in the relevant doping range. The solid blue line is a glue function estimate for Bi2212 from Raman spectroscopy [64], again at optimal doping; results in $B_{1 g}$ and $B_{2 g}$ symmetry are weighted $2: 1$ to best highlight the close correspondence of the energy scales with the RIXS result.

as noted, because the fully-2D-integrated dynamic charge response may indeed be an order of magnitude larger than what we observe in the immediate vicinity of $\boldsymbol{q}_{\mathrm{CDW}}[20,68]$. We also note that up to about $20 \%$ of the nominal paramagnon signal could be dynamic charge correlations [68]. As seen from Fig. 6, there exists an intriguing connection between the charge and magnetic scales obtained from our RIXS experiment and those of the pairing glue function extracted via inversion of optical spectroscopy data for $\mathrm{Hg} 1201$ [63]. Qualitatively similar results have been obtained from Raman scattering, and Fig. 6 includes a result for Bi2212 [64]. Moreover, an analysis of Raman spectra for LSCO revealed a relatively narrow peak in the glue function below $\sim 100 \mathrm{meV}$ and a broad hump up to $\sim 500 \mathrm{meV}$, seen in both $B_{2 g}$ and $B_{1 g}$ symmetry channels [38]. It was concluded that, in $B_{1 g}$ symmetry, spin modes contribute more strongly and at all frequencies, whereas charge modes are particularly prevalent at low or intermediate frequency in the $B_{2 g}$ channel. Tunneling data for optimally doped Bi2212 $\left(T_{c}=95 \mathrm{~K}\right)$, the lowest doping level investigated, indicate a strong local maximum at $\sim 40 \mathrm{meV}$ and weaker, broad feature peaked just above $100 \mathrm{meV}$ [65].
As noted, these spectroscopies yield complex averages over the Brillouin zone and do not distinguish charge from magnetic response. RIXS has a key advantage over these and other techniques, as it is a rather direct, momentumand energy-resolved measure of the charge and magnetic correlations. We emphasize though that RIXS is not a probe of the pairing-glue function, and the comparison in Fig. 6 is by no means quantitative; instead, RIXS complements other spectroscopic probes, as it indicates the nature of the collective excitations at the two energy scales that are likely involved in pairing.

Finally, we point out that a recent RIXS study of electron-doped NCCO suggests a coupling between dynamic magnetic and charge correlations [94]. In holedoped cuprates, on the other hand, there is no clear evidence for such a coupling, except for the special case of the "214" family of materials that exhibit charge-spin stripe order [107]. While we cannot rule out a small (20\% or less) charge contribution to the nominal paramagnon response for underdoped $\mathrm{Hg} 1201$ [68], we find no evidence of a significant coupling: the extracted paramagnon energy (Fig. 4) and damping coefficient (Fig. S4 in Ref. [68]) are insensitive to the dynamic charge correlations, consistent with the fact that the charge and magnetic excitations have somewhat different characteristic energy scales at or near $\boldsymbol{q}_{\mathrm{CDW}}$.

\section{CONCLUSION}

In conclusion, we have measured the charge dynamics and paramagnon response along the $\mathrm{Cu}-\mathrm{O}$ bond direction of underdoped $\mathrm{Hg} 1201$ using RIXS at the $\mathrm{Cu} L_{3}$ edge with high-energy resolution. This has allowed us to discern dynamic correlations from the quasistatic CDW response. Above $T_{\mathrm{CDW}}$, the temperature previously identified with the onset of CDW correlations, the response is purely dynamic, with an energy scale comparable to both the superconducting gap and the low-energy PG. As expected, quasistatic CDW correlations are observed at low temperature. However, there also exists an additional dynamic signature with a remarkably high-energy scale that appears to be an imprint of the high-energy PG and associated with the strong electronic correlations that cause the chargetransfer gap of the undoped parent insulators. It is a distinct possibility that the dynamic correlations identified here have a different underlying physical mechanism than the CDW phenomenon, that they extend through much of the Brillouin zone, and that they significantly contribute to the superconducting pairing glue. At the same time, the various correlations between energy scales identified here are somewhat tentative, and a more detailed examination of doping dependences is needed. The present work sets the foundation for future RIXS measurements of the detailed temperature and doping dependence of the dynamic CDW correlations in the model cuprate $\mathrm{Hg} 1201$ and, more broadly, for efforts to identify the pairing glue and 
understand the CDW phenomenon of the cuprates in the context of large local charge fluctuations.

\section{ACKNOWLEDGMENTS}

We thank the ESRF for the allocation of beam time at ID32, D. Pelc for extensive discussions, and N. Barišić, A. V. Chubukov, G. Ghiringhelli, J. Haase, M.-H. Julien, Yuan Li, D. Pelc, D. Reznik, and E. H. da Silva Neto for valuable comments on the manuscript. The work at University of Minnesota was funded by the Department of Energy through the University of Minnesota Center for Quantum Materials, under Grant No. DE-SC-0016371. The work at the TU Wien was supported by FWF Project No. P27980-N36 and the European Research Council (ERC Consolidator Grant No. 725521). I. B. was supported by the National Science Centre (NCN, Poland) under Grant No. UMO-2019/32/T/ST3/0019.

[1] B. Keimer, S. A. Kivelson, M. R. Norman, S. Uchida, and J. Zaanen, From Quantum Matter to High-Temperature Superconductivity in Copper Oxides, Nature (London) 518, 179 (2015).

[2] J. M. Tranquada, B. J. Sternlieb, J. D. Axe, Y. Nakamura, and S. Uchida, Evidence for Stripe Correlations of Spins and Holes in Copper Oxide Superconductors, Nature (London) 375, 561 (1995).

[3] J. E. Hoffman, E. W. Hudson, K. M. Lang, V. Madhavan, H. Eisaki, S. Uchida, and J. C. Davis, A Four Unit Cell Periodic Pattern of Quasi-Particle States Surrounding Vortex Cores in $\mathrm{Bi}_{2} \mathrm{Sr}_{2} \mathrm{CaCu}_{2} \mathrm{O}_{8+\delta}$, Science 295, 466 (2002).

[4] C. Howald, H. Eisaki, N. Kaneko, M. Greven, and A. Kapitulnik, Periodic Density-of-States Modulations in Superconducting $\mathrm{Bi}_{2} \mathrm{Sr}_{2} \mathrm{CaCu}_{2} \mathrm{O}_{8+\delta}$, Phys. Rev. B 67, 014533 (2003).

[5] T. Wu, H. Mayaffre, S. Krämer, M. Horvatić, C. Berthier, W. N. Hardy, R. Liang, D. A. Bonn, and M.-H. Julien, Magnetic-Field-Induced Charge-Stripe Order in the HighTemperature Superconductor $\mathrm{YBa}_{2} \mathrm{Cu}_{3} \mathrm{O}_{y}, \quad$ Nature (London) 477, 191 (2011).

[6] G. Ghiringhelli, M. Le Tacon, M. Minola, C. Mazzoli, N. B. Brookes, G. M. De Luca, A. Frano, D. G. Hawthorn, F. He, T. Loew, M. M. Sala, D. C. Peets, M. Salluzzo, E. Schierle, R. Sutarto, G. A. Sawatzky, E. Weschke, B. Keimer, and L. Braicovich, Long-Range Incommensurate Charge Fluctuations in $(\mathrm{Y}, \mathrm{Nd}) \mathrm{Ba}_{2} \mathrm{Cu}_{3} \mathrm{O}_{6+\delta}$, Science 337, 821 (2012).

[7] J. Chang, E. Blackburn, A. T. Holmes, N. B. Christensen, J. Larsen, J. Mesot, R. Liang, D. A. Bonn, W. N. Hardy, A. Watenphul, M. v. Zimmermann, E. M. Forgan, and S. M. Hayden, Direct Observation of Competition between Superconductivity and Charge Density Wave Order in $\mathrm{YBa}_{2} \mathrm{Cu}_{3} \mathrm{O}_{6.67}$, Nat. Phys. 8, 871 (2012).

[8] R. Comin, A. Frano, M. M. Yee, Y. Yoshida, H. Eisaki, E. Schierle, E. Weschke, R. Sutarto, F. He, A. Soumyanarayanan, Y. He, M. Le Tacon, I. S. Elfimov,
J. E. Hoffman, G. A. Sawatzky, B. Keimer, and A. Damascelli, Charge Order Driven by Fermi-Arc Instability in $\mathrm{Bi}_{2} \mathrm{Sr}_{2-x} \mathrm{La}_{x} \mathrm{CuO}_{6+\delta}$, Science 343, 390 (2014).

[9] W. Tabis, Y. Li, M. Le Tacon, L. Braicovich, A. Kreyssig, M. Minola, G. Dellea, E. Weschke, M. J. Veit, M. Ramazanoglu, A. I. Goldman, T. Schmitt, G. Ghiringhelli, N. Barišić, M. K. Chan, C. J. Dorow, G. Yu, X. Zhao, B. Keimer, and M. Greven, Charge Order and Its Connection with Fermi-Liquid Charge Transport in a Pristine High- $\mathrm{T}_{c}$ Cuprate, Nat. Commun. 5, 5875 (2014).

[10] T. P. Croft, C. Lester, M. S. Senn, A. Bombardi, and S. M. Hayden, Charge Density Wave Fluctuations in $\mathrm{La}_{2-x} \mathrm{Sr}_{x} \mathrm{CuO}_{4}$ and Their Competition with Superconductivity, Phys. Rev. B 89, 224513 (2014).

[11] M. Hashimoto, G. Ghiringhelli, W. S. Lee, G. Dellea, A. Amorese, C. Mazzoli, K. Kummer, N. B. Brookes, B. Moritz, Y. Yoshida, H. Eisaki, Z. Hussain, T. P. Devereaux, Z. X. Shen, and L. Braicovich, Direct Observation of Bulk Charge Modulations in Optimally Doped $\mathrm{Bi}_{1.5} \mathrm{~Pb}_{0.6} \mathrm{Sr}_{1.54} \mathrm{CaCu}_{2} \mathrm{O}_{8+\delta}$, Phys. Rev. B 89, 220511(R) (2014).

[12] T. Wu, H. Mayaffre, S. Krämer, M. Horvatic, C. Berthier, W. N. Hardy, R. Liang, D. A. Bonn, and M. H. Julien, Incipient Charge Order Observed by NMR in the Normal State of $\mathrm{YBa}_{2} \mathrm{Cu}_{3} \mathrm{O}_{y}$, Nat. Commun. 6, 6438 (2015).

[13] E. H. da Silva Neto, P. Aynajian, A. Frano, R. Comin, E. Schierle, E. Weschke, A. Gyenis, J. Wen, J. Schneeloch, Z. $\mathrm{Xu}, \mathrm{S}$. Ono, G. Gu, M. Le Tacon, and A. Yazdani, Ubiquitous Interplay between Charge Ordering and High-Temperature Superconductivity in Cuprates, Science 343, 393 (2014).

[14] E. H. da Silva Neto, R. Comin, F. He, R. Sutarto, Y. Jiang, R. L. Greene, G. Sawatzky, and A. Damascelli, Charge Ordering in the Electron-Doped Superconductor $\mathrm{Nd}_{2-x} \mathrm{Ce}_{x} \mathrm{CuO}_{4}$, Science 347, 282 (2015).

[15] S. Gerber, H. Jang, H. Nojiri, S. Matsuzawa, H. Yasumura, D. A. Bonn, R. Liang, W. N. Hardy, Z. Islam, A. Mehta, S. Song, M. Sikorski, D. Stefanescu, Y. Feng, S. A. Kivelson, and T. P. Devereaux, Three-Dimensional Charge Density Wave Order in $\mathrm{YBa}_{2} \mathrm{Cu}_{3} \mathrm{O}_{6.67}$ at High Magnetic Fields, Science 350, 949 (2015).

[16] J. Chang, E. Blackburn, O. Ivashko, A. T. Holmes, N. B. Christensen, M. Hücker, R. Liang, D. A. Bonn, W. N. Hardy, U. Rütt, M. V. Zimmermann, E. M. Forgan, and S. M. Hayden, Magnetic Field Controlled Charge Density Wave Coupling in Underdoped $\mathrm{YBa}_{2} \mathrm{Cu}_{3} \mathrm{O}_{6+x}$, Nat. Commun. 7, 11494 (2016).

[17] Y. Y. Peng, M. Salluzzo, X. Sun, A. Ponti, D. Betto, A. M. Ferretti, F. Fumagalli, K. Kummer, M. Le Tacon, X. J. Zhou, N. B. Brookes, L. Braicovich, and G. Ghiringhelli, Direct Observation of Charge Order in Underdoped and Optimally Doped $\mathrm{Bi}_{2}(\mathrm{Sr}, \mathrm{La})_{2} \mathrm{CuO}_{6+\delta}$ by Resonant Inelastic X-Ray Scattering, Phys. Rev. B 94, 184511 (2016).

[18] E. H. da Silva Neto, B. Yu, M. Minola, R. Sutarto, E. Schierle, F. Boschini, M. Zonno, M. Bluschke, J. Higgins, Y. Li, G. Yu, E. Weschke, F. He, M. Le Tacon, R. L. Greene, M. Greven, G. A. Sawatzky, B. Keimer, and A. Damascelli, Doping-Dependent Charge Order Correlations in Electron-Doped Cuprates, Sci. Adv. 2, e1600782 (2016). 
[19] W. Tabis et al., Synchrotron X-Ray Scattering Study of Charge-Density-Wave Order in $\mathrm{HgBa}_{2} \mathrm{CuO}_{4+\delta}$, Phys. Rev. B 96, 134510 (2017).

[20] R. Arpaia, S. Caprara, R. Fumagalli, G. De Vecchi, Y. Y. Peng, E. Andersson, D. Betto, G. M. De Luca, N. B. Brookes, F. Lombardi, M. Salluzzo, L. Braicovich, C. D. Castro, M. Grilli, and G. Ghiringhelli, Dynamical Charge Density Fluctuations Pervading the Phase Diagram of a Cu-Based High- $\mathrm{T}_{c}$ Superconductor, Science 365, 906 (2019).

[21] C. Castellani, C. Di Castro, and M. Grilli, Non-FermiLiquid Behavior and d-Wave Superconductivity Near the Charge-Density-Wave Quantum Critical Point, Z. Phys. B 103, 137 (1996).

[22] S. A. Kivelson, I. P. Bindloss, E. Fradkin, V. Oganesyan, J. M. Tranquada, A. Kapitulnik, and C. Howald, How to Detect Fluctuating Stripes in the High-Temperature Superconductors, Rev. Mod. Phys. 75, 1201 (2003).

[23] S. Caprara, C. Di Castro, G. Seibold, and M. Grilli, Dynamical Charge Density Waves Rule the Phase Diagram of Cuprates, Phys. Rev. B 95, 224511 (2017).

[24] T. Hanaguri, C. Lupien, Y. Kohsaka, D.-H. Lee, M. Azuma, M. Takano, H. Takagi, and J.C. Davis, A 'Checkerboard' Electronic Crystal State in Lightly Hole-Doped $\mathrm{Ca}_{2-x} \mathrm{Na}_{x} \mathrm{CuO}_{2} \mathrm{Cl}_{2}$, Nature (London) 430, 1001 (2004).

[25] K. M. Shen, F. Ronning, D. H. Lu, F. Baumberger, N. J. C. Ingle, W. S. Lee, W. Meevasana, Y. Kohsaka, M. Azuma, M. Takano, H. Takagi, and Z.-X. Shen, Nodal Quasiparticles and Antinodal Charge Ordering in $\mathrm{Ca}_{2-x} \mathrm{Na}_{x} \mathrm{CuO}_{2} \mathrm{Cl}_{2}$, Science 307, 901 (2005).

[26] W. D. Wise, M. C. Boyer, K. Chatterjee, T. Kondo, T. Takeuchi, H. Ikuta, Yayu Wang, and E. W. Hudson, Charge-Density-Wave Origin of Cuprate Checkerboard Visualized by Scanning Tunnelling Microscopy, Nat. Phys. 4, 696 (2008).

[27] P. Abbamonte, A. Rusydi, S. Smadici, G. D. Gu, G. A. Sawatzky, and D. L. Feng, Spatially Modulated Mottness in $\mathrm{La}_{2-x} \mathrm{Ba}_{x} \mathrm{CuO}_{4}$, Nat. Phys. 1, 155 (2005).

[28] J. Q. Lin, H. Miao, D. G. Mazzone, G. D. Gu, A. Nag, A. C. Walters, M. Garcia-Fernandez, A. Barbour, J. Pelliciari, I. Jarrige, M. Oda, K. Kurosawa, N. Momono, K. Zhou, V. Bisogni, X. Liu, and M. P. M. Dean, Nature of the Charge-Density Wave Excitations in Cuprates, arXiv:2001.10312.

[29] D. Pelc, P. Popčević, M. Požek, M. Greven, and N. Barišić, Unusual Behavior of Cuprates Explained by Heterogeneous Charge Localization, Sci. Adv. 5, eaau4538 (2019).

[30] M. H. Hamidian, S. D. Edkins, C. K. Kim, J. C. Davis, A. P. Mackenzie, H. Eisaki, S. Uchida, M. J. Lawler, E. A. Kim, S. Sachdev, and K. Fujita, Atomic-Scale Electronic Structure of the Cuprate d-Symmetry Form Factor Density Wave State, Nat. Phys. 12, 150 (2016).

[31] Y. Caplan, G. Wachtel, and D. Orgad, Long-Range Order and Pinning of Charge-Density Waves in Competition with Superconductivity, Phys. Rev. B 92, 224504 (2015).

[32] L. Wang, B. Yu, R. Jing, X. Luo, J. Zeng, J. Li, I. Bialo, M. Bluschke, Y. Tang, J. Freyermuth, G. Yu, R. Sutarto,
F. He, E. Weschke, W. Tabis, M. Greven, and Y. Li, Doping-Dependent Phonon Anomaly and Charge-Order Phenomena in the $\mathrm{HgBa}_{2} \mathrm{CuO}_{4+\delta}$ and $\mathrm{HgBa}_{2} \mathrm{CaCu}_{2} \mathrm{O}_{6+\delta}$ Superconductors, arXiv:1910.08254.

[33] P. W. Anderson, Is There Glue in Cuprate Superconductors?, Science 316, 1705 (2007).

[34] S. Dal Conte, C. Giannetti, G. Coslovich, F. Cilento, D. Bossini, T. Abebaw, F. Banfi, G. Ferrini, H. Eisaki, M. Greven, A. Damascelli, D. van der Marel, and F. Parmigiani, Disentangling the Electronic and Phononic Glue in a High$\mathrm{T}_{c}$ Superconductor, Science 335, 1600 (2012).

[35] S. Dal Conte et al., Snapshots of the Retarded Interaction of Charge Carriers with Ultrafast Fluctuations in Cuprates, Nat. Phys. 11, 421 (2015).

[36] A. Abanov, A. V. Chubukov, and J. Schmalian, QuantumCritical Theory of the Spin-Fermion Model and Its Application to Cuprates: Normal State Analysis, Adv. Phys. 52, 119 (2003).

[37] D. J. Scalapino, A Common Thread: The Pairing Interaction for Unconventional Superconductors, Rev. Mod. Phys. 84, 1383 (2012).

[38] L. Fanfarillo, M. Mori, M. Campetella, M. Grilli, and S. Caprara, Glue Function of Optimally and Overdoped Cuprates from Inversion of the Raman Spectra, J. Phys. Condens. Matter 28, 065701 (2016).

[39] A. Yamamoto, W.Z. Hu, and S. Tajima, Thermoelectric Power and Resistivity of $\mathrm{HgBa}_{2} \mathrm{CuO}_{4+\delta}$ Over a Wide Doping Range, Phys. Rev. B 63, 024504 (2000).

[40] N. Barišić, M. K. Chan, Y. Li, G. Yu, X. Zhao, M. Dressel, A. Smontara, and M. Greven, Universal Sheet Resistance and Revised Phase Diagram of the Cuprate HighTemperature Superconductors, Proc. Natl. Acad. Sci. U.S.A. 110, 12235 (2013).

[41] N. Barišić, M. K. Chan, M. J. Veit, C. J. Dorow, Y. Ge, Y. Tang, W. Tabis, G. Yu, X. Zhao, and M. Greven, Evidence for a Universal Fermi-Liquid Scattering Rate throughout the Phase Diagram of the Copper-Oxide Superconductors, New J. Phys. 21, 113007 (2019).

[42] S. I. Mirzaei, D. Stricker, J. N. Hancock, C. Berthod, A. Georges, E. van Heumen, M. K. Chan, X. Zhao, Y. Li, M. Greven, N. Barišić, and D. van der Marel, Spectroscopic Evidence for Fermi Liquid-like Energy and Temperature Dependence of the Relaxation Rate in the Pseudogap Phase of the Cuprates, Proc. Natl. Acad. Sci. U.S.A. 110, 5774 (2013).

[43] M. K. Chan, M. J. Veit, C. J. Dorow, Y. Ge, Y. Li, W. Tabis, Y. Tang, X. Zhao, N. Barišić, and M. Greven, In-Plane Magnetoresistance Obeys Kohler's Rule in the Pseudogap Phase of Cuprate Superconductors, Phys. Rev. Lett. 113, 177005 (2014).

[44] J. P. Hinton, E. Thewalt, Z. Alpichshev, F. Mahmood, J. D. Koralek, M. K. Chan, M. J. Veit, C. J. Dorow, N. Barišić, A. F. Kemper, D. A. Bonn, W. N. Hardy, R. Liang, N. Gedik, M. Greven, A. Lanzara, and J. Orenstein, The Rate of Quasiparticle Recombination Probes the Onset of Coherence in Cuprate Superconductors, Sci. Rep. 6, 23610 (2016).

[45] S. Blanco-Canosa, A. Frano, E. Schierle, J. Porras, T. Loew, M. Minola, M. Bluschke, E. Weschke, B. Keimer, 
and M. Le Tacon, Resonant X-Ray Scattering Study of Charge Density Wave Correlations in $\mathrm{YBa}_{2} \mathrm{Cu}_{3} \mathrm{O}_{6+x}$, Phys. Rev. B 90, 054513 (2014).

[46] L. Chaix, G. Ghiringhelli, Y. Y. Peng, M. Hashimoto, B. Moritz, K. Kummer, N. B. Brookes, Y. He, S. Chen, S. Ishida, Y. Yoshida, H. Eisaki, M. Salluzzo, L. Braicovich, Z.-X. Shen, T. P. Devereaux, and W.-S. Lee, Dispersive Charge Density Wave Excitations in $\mathrm{Bi}_{2} \mathrm{Sr}_{2} \mathrm{CaCu}_{2} \mathrm{O}_{8+\delta}$, Nat. Phys. 13, 952 (2017).

[47] D. H. Torchinsky, F. Mahmood, A. T. Bollinger, I. Božović, and N. Gedik, Fluctuating Charge-Density Waves in a Cuprate Superconductor, Nat. Mater. 12, 387 (2013).

[48] J. P. Hinton, J. D. Koralek, Y. M. Lu, A. Vishwanath, J. Orenstein, D. A. Bonn, W. N. Hardy, and R. Liang, New Collective Mode in $\mathrm{YBa}_{2} \mathrm{Cu}_{3} \mathrm{O}_{6+x}$ Observed by TimeDomain Reflectometry, Phys. Rev. B 88, 060508 (2013).

[49] H. Eisaki, N. Kaneko, D. L. Feng, A. Damascelli, P. K. Mang, K. M. Shen, Z.-X. Shen, and M. Greven, Effect of Chemical Inhomogeneity in Bismuth-Based Copper Oxide Superconductors, Phys. Rev. B 69, 064512 (2004).

[50] N. Barišić, Y. Li, X. Zhao, Y.-C. Cho, G. Chabot-Couture, G. Yu, and M. Greven, Demonstrating the Model Nature of the High-Temperature Superconductor $\mathrm{HgBa}_{2} \mathrm{CuO}_{4+\delta}$, Phys. Rev. B 78, 054518 (2008).

[51] Y. Li, N. Egetenmeyer, J. L. Gavilano, N. Barišić, and M. Greven, Magnetic Vortex Lattice in $\mathrm{HgBa}_{2} \mathrm{CuO}_{4+\delta} \mathrm{Ob}$ served by Small-Angle Neutron Scattering, Phys. Rev. B 83, 054507 (2011).

[52] N. Barišić, S. Badoux, M. K. Chan, C. Dorow, W. Tabis, B. Vignolle, G. Yu, J. Béard, X. Zhao, C. Proust, and M. Greven, Universal Quantum Oscillations in the Underdoped Cuprate Superconductors, Nat. Phys. 9, 761 (2013).

[53] M. K. Chan, C. J. Dorow, L. Mangin-Thro, Y. Tang, Y. Ge, M. J. Veit, G. Yu, X. Zhao, A. D. Christianson, J. T. Park, Y. Sidis, P. Steffens, D. L. Abernathy, P. Bourges, and M. Greven, Commensurate Antiferromagnetic Excitations as a Signature of the Pseudogap in the Tetragonal High- $\mathrm{T}_{c}$ Cuprate $\mathrm{HgBa}_{2} \mathrm{CuO}_{4+\delta}$, Nat. Commun. 7, 10819 (2016).

[54] M. K. Chan, N. Harrison, R. D. McDonald, B. J. Ramshaw, K. A. Modic, N. Barišić, and M. Greven, Single Reconstructed Fermi-Surface Pocket in an Underdoped SingleLayer Cuprate Superconductor, Nat. Commun. 7, 12244 (2016).

[55] P. Popčević, D. Pelc, Y. Tang, K. Velebit, Z. Anderson, V. Nagarajan, G. Yu, M. Požek, N. Barišić, and M. Greven, Percolative Nature of the Direct-Current Paraconductivity in Cuprate Superconductors, npj Quantum Mater. 3, 42 (2018).

[56] D. Pelc, M. J. Veit, C. J. Dorow, Y. Ge, N. Barišić, and M. Greven, Resistivity Phase Diagram of Cuprates Revisited, arXiv:1902.00529.

[57] C. Berthier, NMR Study on a $2 \mathrm{H}-\mathrm{NbSe}_{2}$ Single Crystal: A Microscopic Investigation of the Charge Density Waves State, J. Phys. C 11, 797 (1978).

[58] C. J. Arguello, S. P. Chockalingam, E. P. Rosenthal, L. Zhao, C. Gutiérrez, J.H. Kang, W. C. Chung, R. M. Fernandes, S. Jia, A. J. Millis, R. J. Cava, and A. N. Pasupathy, Visualizing the Charge Density Wave
Transition in $2 \mathrm{H}-\mathrm{NbSe}_{2}$ in Real Space, Phys. Rev. B 89, 235115 (2014).

[59] U. Chatterjee, J. Zhao, M. Iavarone, R. Di Capua, J. P. Castellan, G. Karapetrov, C. D. Malliakas, M. G. Kanatzidis, H. Claus, J. P. C. Ruff, F. Weber, J. van Wezel, J.C. Campuzano, R. Osborn, M. Randeria, M. Trivedi, M. R. Norman, and S. Rosenkranz, Emergence of Coherence in the Charge-Density Wave State of $2 \mathrm{H}-\mathrm{NbSe}_{2}$, Nat. Commun. 6 , 6313 (2015).

[60] T. Honma and P.H. Hor, Unified Electronic Phase Diagram for Hole-Doped High- $\mathrm{T}_{c}$ Cuprates, Phys. Rev. B 77, 184520 (2008).

[61] S. V. Dordevic, C. C. Homes, J. J. Tu, T. Valla, M. Strongin, P. D. Johnson, G. D. Gu, and D. N. Basov, Extracting the Electron-Boson Spectral Function $\alpha^{2} F(\omega)$ from Infrared and Photoemission Data Using Inverse Theory, Phys. Rev. B 71, 104529 (2005).

[62] J. Hwang, T. Timusk, E. Schachinger, and J. P. Carbotte, Evolution of the Bosonic Spectral Density of the HighTemperature Superconductor $\mathrm{Bi}_{2} \mathrm{Sr}_{2} \mathrm{CaCu}_{2} \mathrm{O}_{8+\delta}$, Phys. Rev. B 75, 144508 (2007).

[63] E. van Heumen, E. Muhlethaler, A. B. Kuzmenko, H. Eisaki, W. Meevasana, M. Greven, and D. van der Marel, Optical Determination of the Relation between the Electron-Boson Coupling Function and the Critical Temperature in High- $T_{c}$ Cuprates, Phys. Rev. B 79, 184512 (2009).

[64] B. Muschler, W. Prestel, E. Schachinger, J. P. Carbotte, R. Hackl, S. Ono, and Y. Ando, An Electron-Boson Glue Function Derived from Electronic Raman Scattering, J. Phys. Condens. Matter 22, 375702 (2010).

[65] O. Ahmadi, L. Coffey, J. F. Zasadzinski, N. Miyakawa, and L. Ozyuzer, Eliashberg Analysis of Tunneling Experiments: Support for the Pairing Glue Hypothesis in Cuprate Superconductors, Phys. Rev. Lett. 106, 167005 (2011).

[66] Z. Anderson, Y. Tang, V. Nagarajan, M. K. Chan, C. J. Dorow, G. Yu, D. L. Abernathy, A. D. Christianson, L. Mangin-Thro, P. Steffens, Y. Sidis, P. Bourges, and M. Greven (to be published).

[67] N. B. Brookes, F. Yakhou-Harris, K. Kummer, A. Fondacaro, J. C. Cezar, D. Betto, E. Velez-Fort, A. Amorese, G. Ghiringhelli, L. Braicovich, R. Barrett, G. Berruyer, F. Cianciosi, L. Eybert, P. Marion, P. van der Linden, and L. Zhang, The Beamline ID32 at the ESRF for Soft X-Ray High Energy Resolution Resonant Inelastic X-Ray Scattering and Polarisation Dependent X-Ray Absorption Spectroscopy, Nucl. Instrum. Methods Phys. Res. 903, 175 (2018).

[68] See Supplemental Material at http://link.aps.org/ supplemental/10.1103/PhysRevX.10.021059 for additional information regarding experimental details, paramagnon data analysis, polarization dependence, comparison with NBCO, fit to log-normal distribution, energy integration of RIXS data, and analysis of hightemperature CDW response in YBCO.

[69] L. Braicovich, M. M. Sala, L. J. P. Ament, V. Bisogni, M. Minola, G. Balestrino, D. Di Castro, G. M. De Luca, M. Salluzzo, G. Ghiringhelli, and J. van den Brink, Momentum and Polarization Dependence of Single-Magnon Spectral Weight for $\mathrm{Cu} \mathrm{L}_{3}$-Edge Resonant Inelastic 
X-Ray Scattering from Layered Cuprates, Phys. Rev. B 81, 174533 (2010).

[70] M. d'Astuto, A. Mirone, P. Giura, D. Colson, A. Forget, and M. Krisch, Phonon Dispersion in the One-Layer Cuprate $\mathrm{HgBa}_{2} \mathrm{CuO}_{4+\delta}$, J. Phys. Condens. Matter 15, 8827 (2003).

[71] H. Uchiyama, A. Q. R. Baron, S. Tsutsui, Y. Tanaka, W.-Z. $\mathrm{Hu}$, A. Yamamoto, S. Tajima, and Y. Endoh, Softening of $\mathrm{Cu}-\mathrm{O}$ Bond Stretching Phonons in Tetragonal $\mathrm{HgBa}_{2} \mathrm{CuO}_{4+\delta}$, Phys. Rev. Lett. 92, 197005 (2004).

[72] I. Ahmadova, T. C. Sterling, A. C. Sokolik, D. Abernathy, M. Greven, and D. L. Reznik, Phonon Spectrum of Underdoped $\mathrm{HgBa}_{2} \mathrm{CuO}_{4+\delta}$ Investigated by Neutron Scattering, Phys. Rev. B 101, 184508 (2020).

[73] M. W. Haverkort, Theory of Resonant Inelastic X-Ray Scattering by Collective Magnetic Excitations, Phys. Rev. Lett. 105, 167404 (2010).

[74] L. J. P. Ament, G. Ghiringhelli, M. M. Sala, L. Braicovich, and J. van den Brink, Theoretical Demonstration of How the Dispersion of Magnetic Excitations in Cuprate Compounds Can be Determined Using Resonant Inelastic X-Ray Scattering, Phys. Rev. Lett. 103, 117003 (2009).

[75] M. Le Tacon, G. Ghiringhelli, J. Chaloupka, M. M. Sala, V. Hinkov, M. W. Haverkort, M. Minola, M. Bakr, K. J. Zhou, S. Blanco-Canosa, C. Monney, Y. T. Song, G. L. Sun, C. T. Lin, G. M. De Luca, M. Salluzzo, G. Khaliullin, T. Schmitt, L. Braicovich, and B. Keimer, Intense Paramagnon Excitations in a Large Family of HighTemperature Superconductors, Nat. Phys. 7, 725 (2011).

[76] Y. Murakami and S. Ishihara, in Resonant X-Ray Scattering in Correlated Systems, Springer Tracts in Modern Physics (Springer, New York, 2017).

[77] R. Coldea, S. M. Hayden, G. Aeppli, T. G. Perring, C. D. Frost, T. E. Mason, S. W. Cheong, and Z. Fisk, Spin Waves and Electronic Interactions in $\mathrm{La}_{2} \mathrm{CuO}_{4}$, Phys. Rev. Lett. 86, 5377 (2001).

[78] N. S. Headings, S. M. Hayden, R. Coldea, and T. G. Perring, Anomalous High-Energy Spin Excitations in the High- $\mathrm{T}_{c} \quad$ Superconductor Parent Antiferromagnet $\mathrm{La}_{2} \mathrm{CuO}_{4}$, Phys. Rev. Lett. 105, 247001 (2010).

[79] O. Ivashko, N. E. Shaik, X. Lu, C. G. Fatuzzo, M. Dantz, P. G. Freeman, D. E. McNally, D. Destraz, N. B. Christensen, T. Kurosawa, N. Momono, M. Oda, C. E. Matt, C. Monney, H. M. Rønnow, T. Schmitt, and J. Chang, Damped Spin Excitations in a Doped Cuprate Superconductor with Orbital Hybridization, Phys. Rev. B 95, 214508 (2017).

[80] M. P. M. Dean, G. Dellea, R. S. Springell, F. YakhouHarris, K. Kummer, N. B. Brookes, X. Liu, Y-J. Sun, J. Strle, T. Schmitt, L. Braicovich, G. Ghiringhelli, I. Božović, and J. P. Hill, Persistence of Magnetic Excitations in $\mathrm{La}_{2-x} \mathrm{Sr}_{x} \mathrm{CuO}_{4}$ from the Undoped Insulator to the Heavily Overdoped Non-Superconducting Metal, Nat. Mater. 12, 1019 (2013).

[81] W. Kohn and D. Sherrington, Two Kinds of Bosons and Bose Condensates, Rev. Mod. Phys. 42, 1 (1970).

[82] W. J. Padilla, Y. S. Lee, M. Dumm, G. Blumberg, S. Ono, K. Segawa, S. Komiya, Y. Ando, and D. N. Basov, Constant Effective Mass across the Phase Diagram of High-T $T_{c}$ Cuprates, Phys. Rev. B 72, 060511(R) (2005).
[83] S. Ono, S. Komiya, and Y. Ando, Strong Charge Fluctuations Manifested in the High-Temperature Hall Coefficient of High- $T_{c}$ Cuprates, Phys. Rev. B 75, 024515 (2007).

[84] J. W. Alldredge, K. Fujita, H. Eisaki, S. Uchida, and K. McElroy, Universal Disorder in $\mathrm{Bi}_{2} \mathrm{Sr}_{2} \mathrm{CaCu}_{2} \mathrm{O}_{8+x}$, Phys. Rev. B 87, 104520 (2013).

[85] K. K. Gomes, A. N. Pasupathy, A. Pushp, S. Ono, Y. Ando, and A. Yazdani, Visualizing Pair Formation on the Atomic Scale in the High- $T_{c}$ Superconductor $\mathrm{Bi}_{2} \mathrm{Sr}_{2} \mathrm{CaCu}_{2} \mathrm{O}_{8+\delta}$, Nature (London) 447, 569 (2007).

[86] J. L. Tallon, J. G. Story, J. R. Cooper, and J. W. Loram, Locating the Pseudogap Closing Point in the Cuprate Superconductors: Absence of Entrant or Reentrant Behavior, arXiv:1907.12018 [Phys. Rev. B (to be published)].

[87] E. G. Dalla Torre, D. Benjamin, Y. He, D. Dentelski, and E. Demler, Friedel Oscillations as a Probe of Fermionic Quasiparticles, Phys. Rev. B 93, 205117 (2016).

[88] L. Yue, S. Xue, J. Li, W. Hu, A. Barbour, F. Zheng, L. Wang, J. Feng, S. B. Wilkins, C. Mazzoli, R. Comin, and Y. Li, Distinct Fingerprints of Charge Density Waves and Electronic Standing Waves in $\mathrm{ZrTe}_{3}$, Nat. Commun. 11, 98 (2020).

[89] N. Doiron-Leyraud, S. Lepault, O. Cyr-Choiniere, B. Vignolle, G. Grissonnanche, F. Laliberté, J. Chang, N. Barišić, M. K. Chan, L. Ji, X. Zhao, Y. Li, M. Greven, C. Proust, and L. Taillefer, Hall, Seebeck, and Nernst Coefficients of Underdoped $\mathrm{HgBa}_{2} \mathrm{CuO}_{4+\delta}$ : Fermi-Surface Reconstruction in an Archetypal Cuprate Superconductor, Phys. Rev. X 3, 021019 (2013).

[90] J. A. Krumhansl, Fine Scale Mesostructures in Superconducting and Other Materials, Lattice Effects in High- $T_{c}$ Superconductors, Proceedings of the Conference, Santa Fe, New Mexico (1992), https://doi.org/10.1142/ 9789814537575.

[91] Y. Y. Peng, A. A. Husain, M. Mitrano, S. Sun, T. A. Johnson, A. V. Zakrzewski, G. J. MacDougall, A. Barbour, I. Jarrige, V. Bisogni, and P. Abbamonte, Enhanced Electron-Phonon Coupling for Charge-Density-Wave Formation in $\mathrm{La}_{1.8-x} \mathrm{Eu}_{0.2} \mathrm{Sr}_{x} \mathrm{CuO}_{4+\delta}$, arXiv:1910.05526.

[92] D. Reznik, Phonon Anomalies and Synamic Stripes, Physica (Amsterdam) 481C, 75 (2012).

[93] S. R. Park, T. Fukuda, A. Hamann, D. Lamago, L. Pintschovius, M. Fujita, K. Yamada, and D. Reznik, Evidence for a Charge Collective Mode Associated with Superconductivity in Copper Oxides from Neutron and $X$-Ray Scattering Measurements of $\mathrm{La}_{2-x} \mathrm{Sr}_{x} \mathrm{CuO}_{4}$, Phys. Rev. B 89, 020506(R) (2014).

[94] E. H. da Silva Neto, M. Minola, B. Yu, W. Tabis, M. Bluschke, D. Unruh, H. Suzuki, Y. Li, G. Yu, D. Betto, K. Kummer, F. Yakhou, N. B. Brookes, M. Le Tacon, M. Greven, B. Keimer, and A. Damascelli, Coupling between Dynamic Magnetic and Charge-Order Correlations in the Cuprate Superconductor $\mathrm{Nd}_{2-x} \mathrm{Ce}_{x} \mathrm{CuO}_{4}$, Phys. Rev. B 98, 161114(R) (2018).

[95] S. Uchida, T. Ido, H. Takagi, T. Arima, Y. Tokura, and S. Tajima, Optical Spectra of $\mathrm{La}_{2-x} \mathrm{Sr}_{x} \mathrm{CuO}_{4}$ : Effect of Carrier Doping on the Electronic Structure of the $\mathrm{CuO}_{2}$ Plane, Phys. Rev. B 43, 7942 (1991). 
[96] Y. S. Lee, K. Segawa, Z. Q. Li, W. J. Padilla, M. Dumm, S. V. Dordevic, C. C. Homes, Y. Ando, and D. N. Basov, Electrodynamics of the Nodal Metal State in Weakly Doped High- $T_{c}$ Cuprates, Phys. Rev. B 72, 054529 (2005).

[97] Y. Li, W. Tabis, G. Yu, N. Barišić, and M. Greven, Hidden Fermi-Liquid Charge Transport in the Antiferromagnetic Phase of the Electron-Doped Cuprate Superconductors, Phys. Rev. Lett. 117, 197001 (2016).

[98] D. Pelc, M. Vučković, M. S. Grbić, M. Požek, G. Yu, T. Sasagawa, M. Greven, and N. Barišić, Emergence of Superconductivity in the Cuprates via a Universal Percolation Process, Nat. Commun. 9, 4327 (2018).

[99] D. Pelc, Z. Anderson, B. Yu, C. Leighton, and M Greven, Universal Superconducting Precursor in Three Classes of Unconventional Superconductors, Nat. Commun. 10, 2729 (2019).

[100] M. Jurkutat, D. Rybicki, O. P. Sushkov, G. V. M. Williams, A. Erb, and J. Haase, Distribution of Electrons and Holes in Cuprate Superconductors as Determined from ${ }^{17} \mathrm{O}$ and ${ }^{63} \mathrm{Cu}$ Nuclear Magnetic Resonance, Phys. Rev. B 90, 140504(R) (2014).

[101] S. Reichardt, M. Jurukutat, A. Erb, and J. Haase, Charge Variations in Cuprate Superconductors from Nuclear Magnetic Resonance, J. Supercond. Novel Magn. 29, 3017 (2016).

[102] A. A. Husain, M. Mitrano, M. S. Rak, S. Rubeck, B. Uchoa, K. March, C. Dwyer, J. Schneeloch, R. Zhong,
G. D. Gu, and P. Abbamonte, Crossover of Charge Fluctuations across the Strange Metal Phase Diagram, Phys. Rev. X 9, 041062 (2019).

[103] Y. Li, M. Le Tacon, M. Bakr, D. Terrade, D. Manske, R. Hackl, L. Ji, M. K. Chan, N. Barišić, X. Zhao, M. Greven, and B. Keimer, Feedback Effect on High-Energy Magnetic Fluctuations in the Model High-Temperature Superconductor $\mathrm{HgBa}_{2} \mathrm{CuO}_{4+\delta}$ Observed by Electronic Raman Scattering, Phys. Rev. Lett. 108, 227003 (2012).

[104] B. Loret, N. Auvray, Y. Gallais, M. Cazayous, A. Forget, D. Colson, M.-H. Julien, I. Paul, M. Civelli, and A. Sacuto, Intimate Link between Charge Density Wave, Pseudogap and Superconducting Energy Scales in Cuprates, Nat. Phys. 15, 771 (2019).

[105] Y. Wang and A. V. Chubukov, Enhancement of Superconductivity at the Onset of Charge-Density-Wave Order in a Metal, Phys. Rev. B 92, 125108 (2015).

[106] G. A. Thomas, J. Orenstein, D. H. Rapkine, M. Capizzi, A. J. Millis, R. N. Bhatt, L.F. Schneemeyer, and J. V. Waszczak, $\mathrm{Ba}_{2} \mathrm{YCu}_{3} \mathrm{O}_{7-\delta}$ : Electrodynamics of Crystals with High Reflectivity, Phys. Rev. Lett. 61, 1313 (1988).

[107] H. Miao, J. Lorenzana, G. Seibold, Y. Y. Peng, A. Amorese, F. Yakhou-Harris, K. Kummer, N. B. Brookes, R. M. Konik, V. Thampy, G. D. Gu, G. Ghiringhelli, L. Braicovich, and M. P. M. Dean, High-Temperature Charge Density Wave Correlations in $\mathrm{La}_{1.875} \mathrm{Ba}_{0.125} \mathrm{CuO}_{4}$ without Spin-Charge Locking, Proc. Natl. Acad. Sci. U.S.A. 114, 12430 (2017). 